\title{
Modelo dinámico adaptativo para la toma de decisiones sostenibles en el ciclo hidrosocial urbano en México'
}

\author{
Iván Vilchis-Mata², Carlos Félix Garrocho-Rangel ${ }^{3}$ \\ y Carlos Díaz-Delgado ${ }^{4}$
}

\begin{abstract}
RESUMEN
Las ciudades son los motores del desarrollo en el siglo XXI. El factor clave para su supervivencia y desarrollo es el agua. Por tanto, es fundamental estudiar la compleja relación disponibilidad-demanda de agua en las ciudades para impulsar el progreso y bienestar de las sociedades. El modelado y simulación del "Ciclo Hidrosocial Urbano" para el periodo 2015-2030 de la Zona Metropolitana de Toluca (ZMT), corresponde a la interrelación estructural y dinámica del modelo teórico conceptual de oferta y demanda de agua del sistema agua-sociedad inmerso en la "Cuenca Hidrosocial", bajo un enfoque metodológico de modelación dinámica adaptiva. Los escenarios develan tendencias insostenibles, severos impactos y consecuencias que exigen una reflexión profunda. Constituye un insumo indispensable para tomar decisiones estratégicas en la planificación y diseño de políticas públicas de gestión del agua, para lograr un suministro de agua sostenible, resiliente y menos vulnerable en las próximas décadas.
\end{abstract}

Palabras clave: Ciclo hidrosocial urbano, cuenca hidrosocial, modelo dinámico adaptativo, gestión del agua, disponibilidad de agua.

\begin{abstract}
Cities are the engines of development in the 21st century. The key factor for its survival and development is water. Therefore, it is fundamental to study the complex water-availability relation in cities to boost the progress and welfare of societies. The modeling and simulation of the Urban Hydrosocial Cycle for the period 2015-2030 in the Metropolitan Area of Toluca (MAT) corresponds to the structural and dynamic interrelationship of the conceptual model of water supply and demand of the water-society system immersed in the Hydrosocial Basin, under a methodological approach of dynamic adaptive modeling. The scenarios reveal unsustainable tendencies, severe impacts and consequences that require deep reflection. It is an indispensable input for making strategic decisions in the planning and design of public water management policies, to achieve a sustainable, resilient and less vulnerable water supply in the coming decades.
\end{abstract}

Keywords: Urban hydrosocial cycle, hydrosocial basin, adaptive dynamic model, water management, water availability.

Artículo recibido 21 de febrero de 2017, el aceptado el 25 de agosto y corregido el 27 de septiembre de 2017. CONACYT-El Colegio Mexiquense A.C (México). E-mail: ivilchis@cmq.edu.mx

Colegio Mexiquense A.C. (México). E-mail: cfgarrocho@gmail.com

4. Centro Interamericano de Recursos del Agua, Universidad Autónoma del Estado de México (México). E-mail: cdiazd@uaemex.mx 
La sostenibilidad urbana y la capacidad de las ciudades para impulsar el desarrollo dependen de la disponibilidad de agua. Esto ha sido a través de la historia un factor regulador de ocupación territorial de la población y determinante del desarrollo económico y social (Diamond, 1997; 2007). Sin embargo, la compleja relación agua-ciudad enfrenta una crisis de disponibilidad de agua, no tanto por su escasez física, sino por la deficiente gestión de los recursos hídricos (Cruz y Martínez, 2009; Cervantes-Jiménez et al., 2017).

Esto es claro en México, que enfrenta dos paradojas clave en disponibilidad de agua. La paradoja económica del agua es que donde se concentra una gran proporción de población y de actividades económicas (v.g. centro y norte del país) se registra baja disponibilidad natural de agua. La paradoja social del agua es que donde más abunda el recurso (sur-sureste de México), ocurren las mayores catástrofes naturales vinculadas al agua. Moraleja: la disponibilidad del recurso no es suficiente para impulsar la disponibilidad de agua y el desarrollo de las ciudades, sino que se requieren esquemas adecuados para su planeación, manejo y administración (Garrocho, 2013: 355-356).

México afronta un grave problema de abastecimiento de agua potable en las ciudades. Su origen radica en que la demanda de agua debido al crecimiento demográfico y de las actividades, es superior a la oferta, lo que aunado a la deficiente gestión de recursos hídricos deriva en sobreexplotación de acuíferos (Bernardino, 2014). El estado que guarda la relación agua-ciudad corresponde al agotamiento de fuentes locales, incremento en niveles de contaminación, aumento de costos de captación y conducción, generación de conflictos de interés entre usuarios, todo vinculado a una gestión del agua que ha puesto menor énfasis en el manejo de la oferta de agua y otorga mayor importancia a reaccionar a una demanda descontrolada e irracional (Aragón y Córdova, 2009).

Lo anterior cobra mayor relevancia en el marco de los acuerdos de la Cumbre para el DesarroIlo Sostenible 2015, donde los Estados Miembros de la ONU aprobaron la Agenda 2030 que incluye 17 Objetivos de Desarrollo Sostenible (ODS). El agua tiene un posicionamiento estratégico en casi todos los objetivos, pero especialmente en ocho de ellos. ${ }^{5}$ El agua, cuya disponibilidad y calidad está directamente relacionada con la vida y el bienestar de la población, es concebida como un bien público fundamental. Como un activo ecosocial clave por su capacidad para salvaguardar la vida y satisfacer un conjunto de funciones estratégicas en lo económico, social y ambiental. Es decir: para hacer viable el desarrollo sostenible. De ahí la necesidad de establecer mecanismos de planeación integrados (en lo social, natural, administrativo y en tiempo y espacio), para armonizar el valor del agua de acuerdo con sus circunstancias y como prioridad en los problemas de las sociedades (presentes y futuras) (Aguilera Klink, 2006).

En este sentido, el documento se encuentra dividido en siete secciones. La primera señala la importancia de abordar el estudio de las ciudades, el objetivo del estudio, así como los alcances esperados de la modelación del "ciclo hidrosocial urbano".

\footnotetext{
Obj. 1 Poner fin a la pobreza; Obj. 2 Hambre Cero; Obj. 3 Buena salud; Obj. 6 Agua limpia y saneamiento; Obj. 11 Ciudades sostenibles; Obj. 13 Acción climática; Obj. 14 Vida marina; y Obj. 15 Vida en la tierra.
} 
La segunda ancla el "concepto de cuenca hidrosocial" presentando de manera sucinta una visión sistemática de la relación existente entre territorio, agua y sociedad con los procesos de gobernanza y gobernabilidad. Tema clave para comprender y analizar el sistema de planeación, resaltando al agua como el factor crítico del sistema para lograr el desarrollo sostenible de las ciudades.

La tercera aclara los elementos conceptuales claves como son: el "ciclo hidrosocial urbano del agua", la gestión integrada del agua y la modelación dinámica adaptativa, razonando la relación agua-sociedad a través de la configuración y reconfiguración de flujos y procesos sociales, naturales y emergentes, en respuesta a la capacidad de adaptación, resiliencia y trasformación del espacio urbano.

La cuarta aborda el proceso de metropolización en México, donde se resalta y caracteriza la importancia de la Zona Metropolitana de Toluca (ZMT), como área de estudio y de análisis del ciclo hidrosocial urbano, en el contexto de su cuenca hidrosocial.

La quinta corresponde a las precisiones metodológicas, requerimientos y tratamiento de datos de entrada al modelo dinámico adaptativo, con base en la ecuación de balance de masas del sistema urbano de abastecimiento de agua.

La sexta desarrolla el proceso de modelación, en función de la estructuración y disponibilidad de datos correspondientes a las variables propuestas, así como los escenarios, tendencias, impactos, consecuencias y acciones emergentes derivadas de las simulaciones.

Finalmente, la séptima señala a manera de conclusión los aspectos relevantes, el grado de cumplimiento de objetivos y metas planteadas, pormenorizando los principales aportes del trabajo y la propuesta de agenda de investigación, como parte de la integración científica, técnica y política del agua en el ámbito urbano.

\section{Agua: el insumo indispensable de las ciudades impulsoras del desarrollo}

Las ciudades son los motores del desarrollo en el siglo XXI. Un factor vital para su supervivencia y desarrollo es el agua. Por tanto, es fundamental estudiar la compleja relación disponibilidad-demanda de agua en las ciudades para impulsar el progreso y bienestar de las sociedades.

El sistema de planeación de México, lamentablemente, no considera al agua como factor crítico de éxito del desarrollo sostenible. Esto ocasiona interferencias, incoherencias y contradicciones en políticas, metas y objetivos de desarrollo, acentuadas por la desarticulación de competencias administrativas, cuya operatividad y vigencia está determinada por distintos ordenamientos jurídicos federales, estatales y municipales (Bernardino, 2014; Cervantes-Jiménez et al., 2017).

Una forma de mitigar o incluso erradicar estas inconsistencias es adoptar una planificación socionatural, espacial y operativamente integrada de los recursos hídricos. Este sistema de planeación debería tener una visión sistémica y objetivos comunes de largo plazo que orienten la toma de decisiones en y entre los tres niveles de gobierno y los distintos actores presentes en 
el socioecosistema. Un sistema de planeación que cree sinergias en lugar de contradicciones y políticas disfuncionales (Ziccardi, 2004).

Por tanto, el objetivo de este trabajo es construir un modelo del ciclo hidrosocial urbano de la Zona Metropolitana de Toluca (ZMT) con un enfoque metodológico de modelación dinámica adaptiva, que permita: i. Establecer cuál es el estado actual que guarda el balance hídrico derivado de la interacción entre los principales factores y variables que intervienen de manera sistémica en el ciclo hidrosocial urbano; ii. Simular escenarios a partir de las interacciones entre la presión del crecimiento demográfico y las actividades económicas con la disponibilidad de agua; y, iii. Alimentar la toma de decisiones (incluso la instrumentación de acciones emergentes) y el diseño de políticas públicas orientadas a lograr un suministro de agua sostenible, resiliente y menos vulnerable en la ZMT.

\section{Cuenca hidrosocial: una visión sistémica}

La circulación del agua es un proceso social y físico, es decir, un flujo híbrido socionatural. En efecto, en el contexto ambiental mundial el agua representa uno de los temas de mayor trascendencia para el desarrollo sostenible (incluido el desarrollo urbano sostenible), debido a desigualdades de distribución geográfica del recurso y a decisiones políticas y económicas que determinan la relación social con el agua (Larsimont \& Grosso, 2014).

La unidad básica de gestión de los recursos hídricos en México es la Cuenca Hidrológica. ${ }^{6}$ Sin embargo, este trabajo propone adoptar una visión más integral del espacio geográfico, a partir de la unidad socioterritorial denominada "Cuenca Hidrosocial". Aquí retomamos la definición de Durán y Delgadillo (2009: 5) y del Centro Agua (2009), que es analizada por Villagómez-Velázquez et al., (2013: 25). Por tanto, entendemos como un concepto que: 1. Rebasa "espacialmente la cuenca hidrográfica", pues además de considerar las zonas de producción y aprovechamiento de agua, contempla también los eventuales trasvases de aguas de cuencas aledañas; 2 . Sobrepasa "jurisdicciones político-administrativas" (v.g. departamento, provincia, estado, municipio); 3. "Se fundamenta en redes que se crean en torno al agua entre usuarios de las zonas altas y zonas bajas" (v.g. redes socio-hídricas) a lo largo de procesos de desarrollo hídrico (v.g. iniciativas locales, intervenciones); 4. "Marca historias particulares que configuran esa cuenca hidrosocial"; 5. 2 Genera equilibrios inestables sujetos a situaciones de conflicto y resolución continuos, donde la cuenca hidrosocial2 puede contraerse o expandirse en el tiempo; 6. Es "limitada tanto por factores físicos" (v.g. fuentes de agua, infraestructura de aprovechamiento, áreas de influencia de los sistemas, etc.) "como sociales" (acuerdos entre usuarios) y "político-administrativos" dado que articula espacios jurisdiccionales y administrativos con problemas y demandas concretas para resolver cuestiones hídricas?

\footnotetext{
Definida como una unidad territorial autónoma, delimitada por un parteaguas, donde el agua se almacena y fluye a través de una red hidrográfica, en coexistencia con otros acuíferos y recursos naturales (LAN, 2016).

El concepto de "Cuenca Hidrosocial" se vincula con el de "Territorio Hidrosocial", tal como lo define Damonte-Valencia (2015: 115): "como la articulación de los espacios físicos de cuenca (incluyendo infraestructura y sistemas hídricos), los espacios sociales (definidos a partir de los usos y manejos materiales y simbólicos que los actores sociales hacen del agua en la cuenca) y los espacios político-administrativos (generados a partir de los discursos de desarrollo territorial y de la institucionalidad de regulación hídrica). Esta articulación se produce en el contexto de interacciones sociopolíticas".
} 
La "Cuenca Hidrosocial" permite comprender y analizar mejor las "externalidades" derivadas de interdepencias socionaturales entre lo hídrico y lo social. Es decir, entre el agua y el sistema de gobernanza del agua, acciones estratégicas ante problemas críticos, definición de responsabilidades de diversos actores y facilitación en la toma de decisiones, lo que en conjunto afecta la coherencia de los procesos de planificación, desarrollo, proyecciones futuras de disponibilidad e implicaciones de la gestión del agua.

Es oportuno aclarar la diferencia, al menos en este trabajo, entre gobernanza y gobernabilidad en materia de recursos hídricos. En política del agua, la gobernanza se refiere a los "procesos y mecanismos de interacción" entre actores gubernamentales y no gubernamentales, como un concepto dinámico de definición, orientación e instrumentación de políticas públicas sobre la estructura actual de gestión del agua, a través de las que opera la sociedad. En cambio, la gobernabilidad se refiere al "estado resultante" de la aplicación de los procesos y mecanismos de interacción, como un proceso democrático administrativo (Domínguez, 2012). Para mejorar la gobernanza y gobernabilidad del agua es necesario conocer y analizar la dimensión espacial de la política del agua.

\section{La relación agua-sociedad: configuración y reconfiguración del espacio urbano}

\section{Ciclo Hidrosocial Urbano del Agua}

El estudio de la ciudad como un socioecosistema permite el análisis de flujos de naturaleza diversa (e.g. energía, bienes, transporte, personas, información). Es decir, de las interacciones entre elementos humanos y no humanos del sistema, de manera tal, que la complejidad de los sistemas urbanos es capturada e interpretada para su análisis y formulación de estrategias de gestión (Batty, 2013; Grimm et al., 2000; Díaz-Delgado et al., 2014).

Abel Wolman (1965) desarrolló el concepto de "metabolismo urbano", en respuesta a la rápida expansión urbana y deterioro en la calidad del aire y del agua en ciudades norteamericanas. Este concepto se apoya en la cuantificación de entradas, salidas y almacenamientos de energía, agua, nutrientes, materiales y residuos de una región urbana, como enfoque adaptativo de soluciones tecnológicas, socio-políticas y manejo de consecuencias. El metabolismo urbano es reconocido como un concepto importante para avanzar en el desarrollo urbano sostenible. Entre todos sus componentes el agua tiene un carácter preeminente (Kennedy et al., 2007; 2011).

En los últimos años se ha empleado el concepto de "metabolismo urbano" en una amplia gama de disciplinas, particularmente de la ecología urbana. Ampliando el conocimiento sobre el uso de los recursos en las ciudades y la comprensión de la interrelación entre las economías urbanas y el ambiente. El resultado: una perspectiva crítica sobre la forma en que lo urbano es producto de las relaciones entre los procesos sociales y naturales (Broto et a.l, 2011). ${ }^{8}$

En los procesos sociales se incluye lo económico, lo cultural, lo político, entre otras muchas dimensiones de "lo social". 
La compleja relación agua-sociedad en espacios urbanos es ampliamente reconocida. Por ello, en los últimos 15 años ha llamado la atención de diversas disciplinas de las ciencias sociales, generando el enfoque del "ciclo hidrosocial" (Figura No 1) que intenta entender cómo el flujo del agua influye en el espacio urbano y cómo se inserta de manera fundamental en la dimensión social de la ciudad (Budds, 2008, 2009; Kaika, 2005; Linton, 2008, 2010; Ridolfi, 2014). ${ }^{9}$ Aquí retomamos la definición de Linton y Budds (2014: 6): "...el 'ciclo hidrosocial' es un proceso socio-natural mediante el que el agua y la sociedad se configuran y reconfiguran mutuamente en el espacio y el tiempo"10.

Figura $\mathrm{N}^{\circ} 1$

Esquema del ciclo hidrosocial

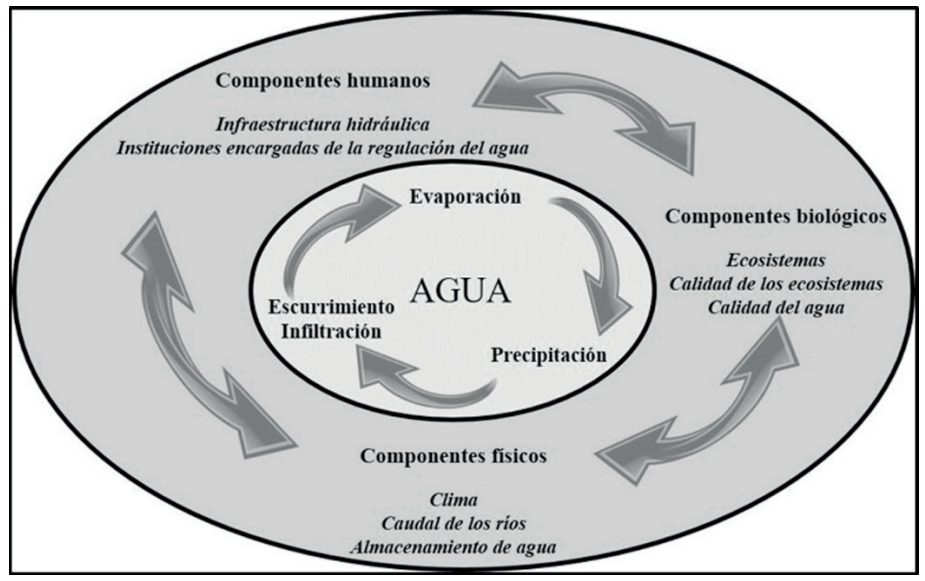

Fuente: Modificado de Rés-EAUx".

La conceptualización de "cuenca hidrosocial" y "ciclo hidrosocial", como medio para teorizar y analizar la relación agua-sociedad, se basan en el concepto del ciclo hidrológico, pero lo modifican de manera importante. Así, mientras el ciclo hidrológico tiende a separar el agua de su contexto social, el "ciclo hidrosocial" asiste deliberadamente a la naturaleza social y política del agua (Linton y Budds, 2014). Esta noción hace referencia a la permanente interacción entre el ciclo hidrológico y el devenir social: la manera compleja y diacrónica en que la acción humana reconfigura el ciclo natural del agua y viceversa (Budds, 2008, 2012; Banister, 2014; Ridolfi, 2014).

El "ciclo hidrosocial" se debe entender como la suma de interrelaciones humanas y físicas relacionadas al agua en las ciudades (Keil \& Young, 2001). Es un intento de mostrar cómo los sistemas sociales, políticos, culturales y económicos regulan el flujo de agua a través de procesos sociales

El concepto del "ciclo hidrosocial" se origina en la ecología política del agua, en un estudio urbano sobre el abastecimiento de agua (Swyngedouw, 2004). Desarrollado a mayor profundidad por Linton (2010) y se vincula con el análisis crítico sobre la sobreexplotación de aguas subterráneas (Budds, 2012)

10 Traducción de los autores. En el trabajo de Linton y Budds (2014: 6) dice textual: "We define the hydrosocial cycle as a socio-natural process by which water and society make and remake each other over space and time".

Red de Estudios e Intercambio en Ciencias Sociales sobre el Aagua, Universidad de Paris X Nanterre. 
(Swyngedouw 2004, 2005; Bakker, 2005; Swyngedouw et al., 2002), reconociendo que, simultáneamente, el agua también moldea la conformación de la sociedad (Wittfogel, 1957).

El "ciclo hidrosocial" plantea una ciencia, cuyo campo es definido entre lo hidrológico y lo social, y, por tanto, un medio de producción de conocimiento crítico sobre la naturaleza social del agua (Maidment, 1993. citado en Linton, 2010:231). De esta manera, además de examinar cómo el agua fluye dentro del ambiente físico (v.g. atmósfera, superficie, subsuelo, biomasa), el "ciclo hidrosocial" también considera cómo el agua es manipulada por los agentes sociales y las instituciones, a través de múltiples instrumentos: obras hidráulicas, legislación, instituciones, prácticas culturales y significados simbólicos (Budds e Hinojosa, 2012).

Paralelamente al concepto de "ciclo hidrosocial", el término waterscape se ha difundido en los estudios sobre el agua, particularmente en los que se derivan del enfoque de "hidropolítica" (Larsimont \& Grosso, 2014:35). El waterscape representa, tanto una unidad de paisaje analizada por sus recursos hídricos, como la interconectividad entre lo hidrológico, lo socio-político y ecosistémico (Molle, 2012). Considera los componentes natural y social del agua, pero subraya sus relaciones y procesos histórico-geográficos (Swyngedouw, 1999). En otras palabras, representa el medio donde se desarrolla el "ciclo hidrosocial".

Las circulaciones entrelazadas del agua, capital y poder, reflejan modos de control, apropiación y gestión de externalidades derivadas del agua (v.g. escasez, inundaciones, contaminación, degradación ambiental). Por tanto, configuran y reconfiguran paisajes cambiantes, fluidos y dinámicos. Un mismo waterscape refleja una fuerte fragmentación territorial entre aguas arriba (upstream) y aguas abajo (downstream) dentro de una misma cuenca hidrográfica, así como también el acceso diferenciado de agua potable en un ámbito urbano (Larsimont \& Grosso, 2014:36).

En el contexto de "cuenca hidrosocial", el concepto de "territorio hidrosocial" conecta con el de paisajes del agua (waterscapes), ya que son entidades espaciales configuradas por flujos físicos de agua, normas de acceso, tecnologías, instituciones, prácticas, discursos y significados que producen y están producidos por relaciones de poder (Budds e Hinojosa, 2012). Los territorios hidrosociales se forman a través de procesos socioecológicos a diversas escalas temporales y espaciales, que frecuentemente no se circunscriben a la realidad fisiográfica de la cuenca hidrográfica (Swyngedouw, 1999).

En síntesis, cualquier modelo del "ciclo hidrosocial" urbano debe considerar los principales procesos socio-naturales, mediante los que el agua y la sociedad se configuran y reconfiguran mutuamente en el espacio y el tiempo.

\section{Gestión Integrada del Agua}

En México más de la mitad de la población vive en ciudades que no ofrecen garantía de suministro de agua potable, saneamiento y tratamiento de aguas residuales (Garrocho, 2013). La contradicción es evidente: el agua es esencial para la vida y prosperidad de la población y sus ciudades. Esto, más las crecientes presiones del cambio climático, el crecimiento demográfico y la urbanización sostenida, han urgido una estrategia nacional (ver el PND 2013-2018) alineada a la visión internacional que subraya que el desarrollo sostenible no se logrará en un mundo sin 
"seguridad hídrica", fundamental para promover el desarrollo y elevar los niveles de vida, especialmente de la población pobre que es la que más se beneficia de la buena gobernabilidad del agua (GWP, 2013).

El marco legal y el sistema de planeación del país subrayan la importancia vital del agua. La Ley de Aguas Nacionales establece que la preservación en cantidad, calidad y sostenibilidad del agua es tarea fundamental del Estado y la Sociedad, y asunto de seguridad nacional (LAN, 2016). En consonancia, el Plan Nacional de Desarrollo 2013-2018 propone enfocar la acción del Estado, garantizar el ejercicio de los derechos sociales a través del acceso al agua potable, y otros servicios básicos, para que los ciudadanos puedan desarrollarse plenamente como individuos y cerrar las brechas de desigualdad social, con el fin de que el país se fundamente en la equidad, cohesión social e igualdad sustantiva (PND 2013-2018). Esto es clave en un país fragmentado y desigual, donde las ciudades, independientemente de su tamaño, manifiestan patrones de crecimiento disfuncionales, excluyentes y vulnerables. Es decir: simplemente insostenibles (Sobrino et al., 2015). Algo similar ocurre en América Latina (Banco Mundial, 2012).

En el discurso, el Estado Mexicano reconoce cuatro temas claves: i. El derecho humano al agua; ii. La importancia del desarrollo, sostenibilidad y equidad social; iii. La necesidad de reconceptualizar las políticas públicas; y, iv. Que el diseño gestión, implementación y monitoreo de las políticas hídricas se instrumentan de manera aislada lo que reduce notablemente su eficacia.

Por tanto, se requiere un enfoque de Gestión Integrada de los Recursos Hídricos (GIRH), entendida como un proceso que promueve la gestión y el desarrollo coordinados del agua, el suelo y otros recursos relacionados, con el fin de maximizar los resultados económicos y el bienestar social de forma equitativa sin comprometer la sostenibilidad de los ecosistemas vitales (Hassing et al., 2009). Las políticas públicas, la gestión de cuencas, el suministro de agua y la planificación urbana ya no pueden ser abordados de manera aislada si verdaderamente se pretende evitar futuros conflictos sociales en torno al agua.

Esto implica, entre otras cosas, repensar paradigmas y contar con instrumentos de planeación que informen científicamente la toma de decisiones, especialmente en ciudades de crecimiento acelerado (Ortiz et al., 2013). Justo en ese nicho encuentra su lugar el modelo dinámico adaptativo del "ciclo hidrosocial urbano" de la ZMT que proponemos en este trabajo.

\section{Modelos Dinámicos Adaptativos}

Los Modelos Dinámicos Adaptativos (MDA) se apoyan en el enfoque "sistémico" (Teoría General de los Sistemas: Von Bertalanffy, 1972), en el del "Ciclo Adaptativo" (Holling, 1986) y en la "Ciencia de la Complejidad" (Johnson, 2011). De manera sucinta, un sistema se define como un conjunto de elementos interrelacionados. Por su parte, el "Ciclo Adaptativo" consta de cuatro fases que ocurren de manera común en los procesos de cambio de los sistemas complejos, como resultado de su dinámica interna e influencia externa: crecimiento $(r)$, conservación (K), liberación o destrucción creativa $(\Omega)$ y reorganización $(\alpha)$ (Figura $\left.N^{\circ} 2\right)$. La fase $(r)$ se caracteriza por la disponibilidad de recursos, estructura de acumulación y alta resiliencia, mientras que en la fase $(K)$ el sistema se interconecta más, es menos flexible y más vulnerable a perturbaciones externas. Estas dos fases ( $r$ y $K)$, pueden interpretarse como una sucesión ecológica donde se organizan 
modos de desarrollo social. En la fase $(\Omega)$ se libera la conectividad del sistema por agentes perturbadores, y en la fase $(\alpha)$ se presenta la innovación y restructuración de procesos económicos y políticos de trasformación social, y se da origen a una nueva fase ( $r$ ), lo que constituye una retroalimentación (o feedback).

Figura $\mathrm{N}^{\circ} 2$

Fases del ciclo adaptativo

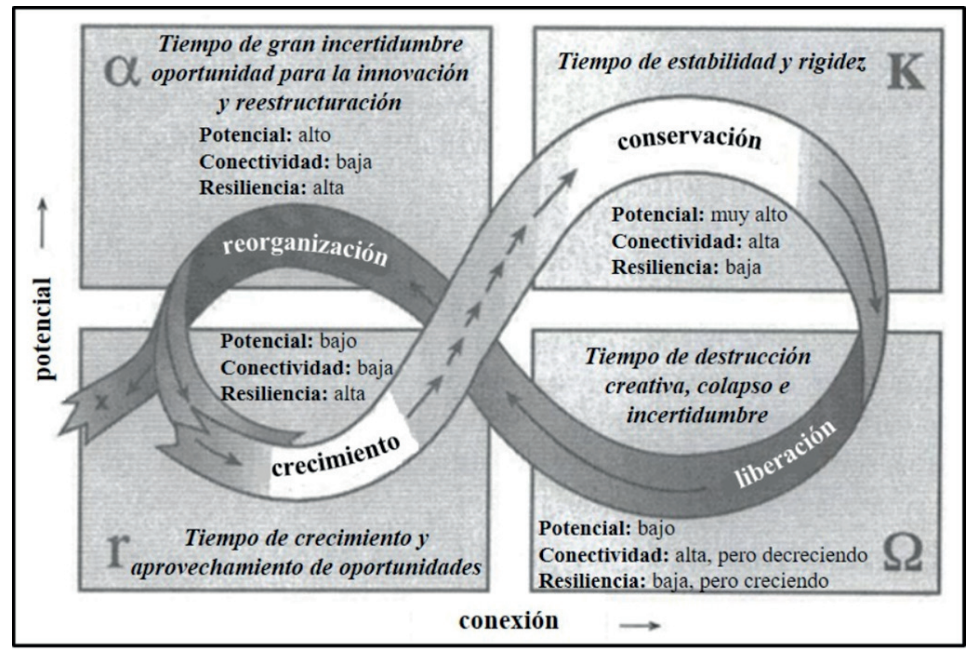

Fuente: Modificado de Castillo y Velázquez (2015).

Finalmente, la Ciencia de la Complejidad estudia fenómenos que emergen de una colección de objetos que interactúan, con un enfoque interdisciplinario: sistemas de comportamiento complejo (Castillo y Velázquez, 2015). Construye modelos predictivos/informativos que consideran la existencia del azar, la indeterminación o la auto-organización. Tiene aplicación tanto en ciencias de la materia, de la salud y en las ciencias sociales (Johnson, 2011). Una característica esencial de los sistemas complejos son las "propiedades emergentes" que surgen de las interacciones del conjunto de componentes sin que pueda ser atribuible a un componente en particular. Esto es, característica que no poseen los componentes individualmente. La complejidad de los sistemas lleva a condiciones de incertidumbre. A su vez, la auto-organización hace referencia a una emergencia de comportamiento colaborativo por supervivencia entre los elementos del sistema. En el caso de sistemas hídricos, se ha citado la reutilización del agua como una propiedad emergente y en el caso del sistema-ciudad un ejemplo de propiedad emergente es la resiliencia urbana, entendida como la habilidad del sistema para absorber cambios y perturbaciones (Castillo y Velázquez, 2015; Ficot, 2009)12.

\footnotetext{
Con particular énfasis en la capacidad de renovación, reorganización y desarrollo de interacciones entre sus componentes. La resiliencia requiere distintos enfoques para explicar la relación dinámica, uno de ellos corresponde a los sistemas complejos adaptativos, al permitir incorporar elementos no estudiados y que permiten exhibir características de dependencia histórica socio-política, cambios discontinuos, no linealidad y múltiples equilibrios, orientados a las ciudades y sociedad, a escenarios de menor vulnerabilidad social (Constas et al., 2014; Weichselgartner y Kelman, 2015).
} 
Raskin (2006), señala que la posibilidad de interacciones, tanto verticales como horizontales, realimentaciones y amplificación en los sistemas socioecológicos (como el "ciclo hidrosocial urbano") puede llevar al sistema a un estado de crisis. Por eso, al considerar los procesos de cambio en los sistemas complejos es importante distinguir entre "adaptación y transformación". La primera se entiende como las alteraciones de las relaciones sociales y de la sociedad con la esfera ecológica, pero manteniendo su estructura. La segunda, puede implicar alteraciones de las relaciones sociales, pero como consecuencia de mutaciones de las estructuras sociales.

Cuando se presenta un impacto o factor de estrés, entonces el sistema entra en una etapa de inestabilidad que conduce a procesos de cambio. El enfoque de sistemas complejos adaptativos asume que los sistemas sociales y sistemas ecológicos son interdependientes y no lineales, con retroalimentaciones en diferentes niveles que permiten al sistema auto-organizarse, adaptarse continuamente y cambiar de una manera impredecible. Por ello surge la necesidad de entender la estructura y los patrones de las interacciones intra e inter subsistemas para explorar e incrementar su resiliencia y capacidad de adaptación y transformación (Castillo y Velázquez, 2015).

Los sistemas complejos adaptativos buscan pautas, interaccionan con el entorno, aprenden de la experiencia y como resultado se adaptan. Las adaptaciones son un comportamiento determinista, las auto-organizaciones son comportamientos aleatorios, resultados positivos de crisis y del aprovechamiento de oportunidades generalmente autogeneradas por el sistema (Cardona, 2001).

La modelación de sistemas dinámicos adaptivos es una herramienta útil para la creación de modelos de simulación de sistemas complejos, que permiten el diseño de escenarios compuestos por diversas estrategias, y la valoración de sus resultados en el sistema (Martínez y Vargas, 2016). El enfoque de sistemas y la modelación dinámica aplicada a la gestión del agua es uno de los campos de investigación y desarrollo tecnológico más activos en las últimas décadas (Giacomoni et al., 2013; Ficot, 2009).

El "ciclo hidrosocial urbano" del agua exhibe la conducta de un sistema complejo: es adaptativo y dinámico. Es decir, se trata de un sistema complejo que no responde a razonamientos lineales causa-efecto, debido básicamente a que existen ciclos de realimentación entre la dinámica poblacional y la disponibilidad de agua (Martínez y Vargas, 2016) ${ }^{13}$.

\section{El proceso de metropolización en México}

\section{Agua en la ciudad y el contexto estratégico de la metropolización}

México es un país en proceso de metropolización (Garrocho, 2013). Las zonas metropolitanas (ZM) son ciudades conformadas por dos o más municipios donde se localiza un asentamiento de

\footnotetext{
La modelización se considera multifacética o perspectivista, porque el modelo que se construye de un sistema real depende del objetivo del modelador (Zeigler, 1984). En este sentido, el objetivo de construir un modelo como una representación simplificada de la estructura y comportamiento de un sistema real (v.g. variables y relaciones funcionales).
} 
50 mil o más habitantes (SEDESOL et al., 2010). Es decir, son ciudades conducidas por varios gobiernos, que en México usualmente están descoordinados. Por esta razón las ZM mexicanas son unidades complejas en su funcionamiento y gestión del agua.

En 2017 se reconocen 59 ZM en México, donde residen 63.8 millones de habitantes: alrededor de 56.8 por ciento de la población nacional (CONAPO, 2011). Es decir, más de la mitad de la población del país vive y trabaja en ciudades con gobiernos descoordinados. De las 59 ZM resaltan 11 que sobrepasan el millón de habitantes ${ }^{14}$. Estas ZM son los motores claves del desarrollo nacional y concentran poco más del 36 por ciento de la población. Se espera que el número de ZM con más de un millón de habitantes se duplique para 2030 (Sobrino et al., 2015). El fenómeno metropolitano impulsará el grado de urbanización en México que pasará de $72 \%$ de población urbana en 2010 a 83\% en 2030 (CONAPO, 2011).

La descoordinación de los gobiernos locales de las ZM y la ausencia de gobiernos o acuerdos metropolitanos que guíen el desarrollo de las ciudades estratégicas del país es una barrera muy seria para la adecuada gestión del agua. La ZM de Toluca (ZMT) no es la excepción: registra descoordinación entre los municipios que la integran y persiste falta de voluntad y obligatoriedad para operar y dar seguimiento a los planes de desarrollo urbano y de política económica y social, incluidos los temas asociados al agua (Martínez y Castillo, 2016:33). Más allá de declaraciones políticas y diagnósticos que no modifican la realidad, lo que se requiere son esquemas formales de planeación metropolitana para establecer directrices, políticas y estrategias, coordinar la ejecución de acciones y contar con instrumentos e indicadores de planeación que permitan guiar ordenadamente el desarrollo integral de la $\mathrm{ZM}^{15}$.

Lo anterior demuestra la importancia de construir un modelo conceptual del "ciclo hidrosocial urbano" de la ZMT. Este modelo debería establecer cuál es el estado actual que guarda el balance hídrico derivado de la interacción de factores y variables que intervienen de manera sistémica en la ZM, y permitir la construcción de escenarios para anticipar consecuencias y alternativas de solución al tema del agua en la "cuenca hidrosocial" de la ZMT.

\section{Caracterización de la Zona Metropolitana de Toluca}

La ZMT alojaba en 2015 a 2.116.506 habitantes (13.1 por ciento de la población total del Estado de México), es la quinta más poblada de México y está conformada por quince municipios (SEDESOL et al., 2010). ${ }^{16}$ Se localiza en el centro-oeste del Estado de México (Figura № 3), en la subprovincia de Lagos y Volcanes de Anáhuac, perteneciente a la provincia del Eje Neovolcánico, constituida por sistemas de topoformas de sierras volcánicas correspondientes al Nevado de Toluca (4.680 msnm) y Sierra de las Cruces. Esta región presenta un paisaje abrupto de lavas

\footnotetext{
La distribución de la población en las principales zonas metropolitanas es aproximadamente la siguiente: más de 20 millones para el Valle de México, 4.4 Guadalajara, 4.1 Monterrey, 2.7 Puebla-Tlaxcala, 1.9 Toluca, 1.7 Tijuana, 1.6 León, 1.3 Juárez-El Paso, 1.2 La Laguna, 1.1 Querétaro y 1.0 San Luis Potosí (CONAPO, 2011).

15 El Valle de Toluca, conocido por su riqueza agrícola y las ciénagas características de su complejidad ecosistémica, donde nació y se desarrolló una ciudad dentro y rodeada de agua en el CARL, ahora sufre de escasez de agua y tiene que importarla del Sistema Cutzamala y exportarla al Valle de México (Gutiérrez y Maderey, 2013).

16 La población en 2015 de los municipios metropolitanos es la siguiente: Almoloya de Juárez 176 mil habitantes, Calimaya 56.000, Chapultepec 12.000, Lerma 147.000, Metepec 228.000, Mexicaltzingo 13.000, Ocoyoacac 66.000, Otzolotepec 84.000, Santa María Rayón 13.000, San Antonio La Isla 27.000, San Mateo Atenco 75.000, Temoaya 104.000, Toluca 874.000, Xonacatlán 52.000 y Zinacantepec 189.000.
} 
dominantes de tipo andesítico, destacan relieves de llanuras y lomeríos enmarcados en el Curso Alto del río Lerma (CARL), que posee rasgos característicos de un vaso lacustre, con una altitud de 2600 msnm, con predominio en las zonas bajas de lomeríos y llanuras, de un clima C(w2)(w) b(i)g templando-subhúmedo, en las porciones de lomeríos y sierras se presenta un clima $C(E)(W 2)$ (w)b(i)g semifrío-subhúmedo y en las partes más elevadas superiores a los $4.000 \mathrm{msnm}$, un clima $\mathrm{E}(\mathrm{T}) \mathrm{H}$ frío, la temperatura media anual es de $14,7^{\circ} \mathrm{C}$, con una precipitación media anual de 900 mm (Hernández, 2014).

Figura $\mathrm{N}^{\circ} 3$

Ubicación de la Zona Metropolitana de Toluca

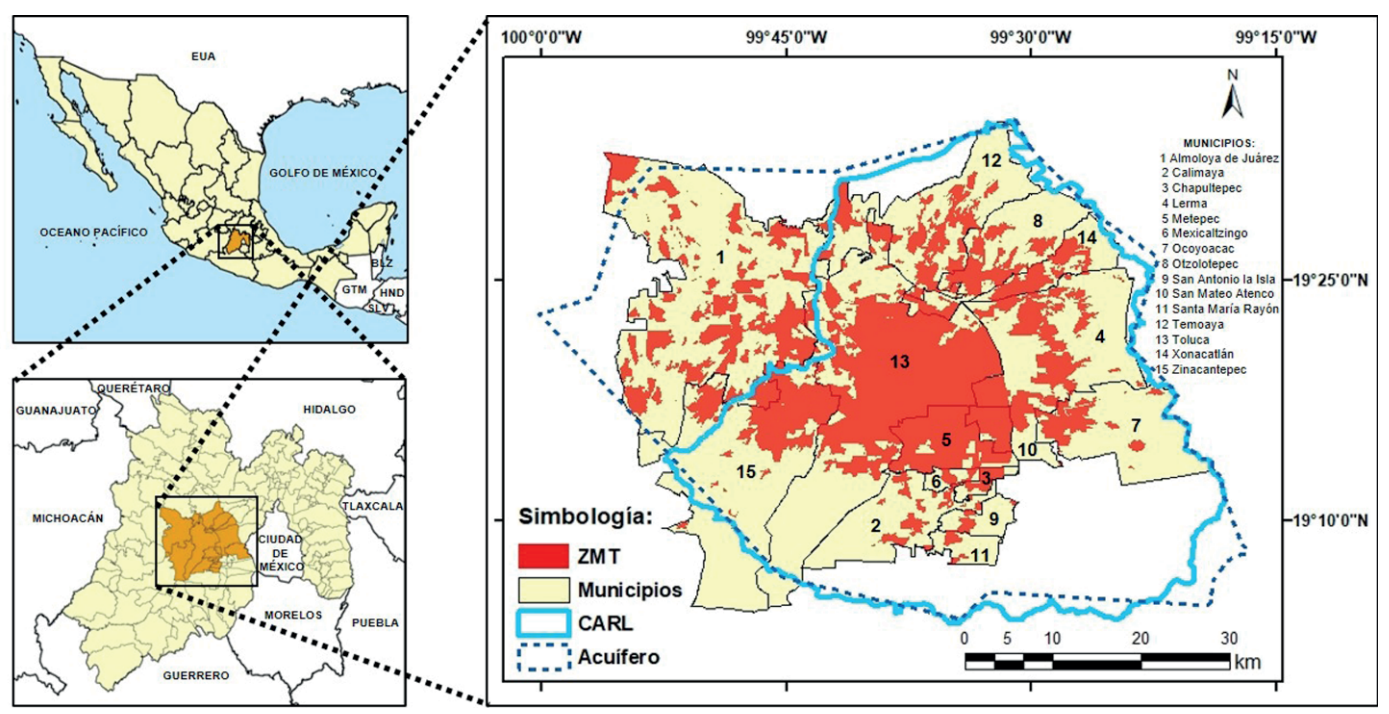

Fuente: Elaboración propia.

La ZMT cuenta con dos fuentes de abastecimiento de agua. La primera corresponde a la extracción por medio de 84 pozos profundos del acuífero de Toluca, que se encuentra en veda para nuevos aprovechamientos desde 1965 debido a su disponibilidad escasa y su condición geohidrológica sobreexplotada (Cuadro $N^{\circ} 1$ ). La segunda fuente de abastecimiento es la importación de agua del Sistema Cutzamala. Este complejo hídrico de producción, almacenamiento, conducción, potabilización y distribución de agua, que suministra el $24 \%$ del agua potable a la red de abastecimiento de 13 delegaciones de la Ciudad de México y 14 municipios del Estado de México, incluye a tres de la ZMT (Ocoyoacac, Lerma y Toluca). Ha estado en funcionamiento durante cerca de 80 años, en sus inicios como parte del Sistema Hidroeléctrico Miguel Alemán, y a partir de 1982, con propósitos de abastecimiento de agua potable (Banco Mundial, 2015).

Es importante señalar, que, aunado a la extracción de agua subterránea, la alteración de los patrones originales de flujo hidrológico, han degradado de manera importante el CARL. Donde los ríos y cauces naturales, se han convertido en parte del drenaje urbano, debido al proceso de urbanización y desarrollo industrial de la ZMT. El cauce del río Lerma es actualmente el principal receptor de las descargas residuales urbanas, incluyendo las de corredores y parques industria- 
Cuadro $\mathrm{N}^{\circ} 1$

Disponibilidad media anual de agua subterránea

\begin{tabular}{|l|c|r|r|r|r|r|r|}
\hline \multicolumn{7}{|c|}{ DXIV Región Hidrológico-Administrativa “Lerma-Santiago-Pacífico" } \\
\hline \multirow{2}{*}{ Clave } & \multirow{2}{*}{ Acuífero } & $\mathrm{R}$ & DNCOM & VCAS & VEXTET & DAS & Déficit \\
\cline { 3 - 8 } & & \multicolumn{6}{|c|}{ Cifras en millones de metros cúbicos anuales } \\
\hline 1501 & Valle de Toluca & 336,8 & 53,6 & 419,9 & 422,4 & 0,0 & $-136,8$ \\
\hline
\end{tabular}

R: recarga media anual; DNCOM: descarga natural comprometida; VCAS: volumen concesionado de agua subterránea; VEXTET: volumen de extracción de agua subterránea consignado en estudios técnicos; DAS disponibilidad media anual de agua subterránea.

Fuente: Actualización de la Disponibilidad Media Anual de Agua Subterránea, publicado en el Diario Oficial de la Federación el 20 de abril 2015.

les, lo que ha derivado en un alto deterioro de la calidad del agua (Esteller y Díaz-Delgado, 2002; Díaz-Delgado et al., 2014).

Esta transformación del medio natural del CARL, ha estado subordinada al interés económico desde la época de la Colonia, donde la producción de maíz fue remplazada por la ganadería. Sin embargo, a mediados del siglo XX, se presenta un cambio drástico en el sistema lacustre con la desecación de las lagunas del Lerma, ocasionada por la disminución del nivel freático, alcanzando un nivel crítico entre 1970 y 1980 con la consolidación del proceso de industrialización. Consecuencias de lo anterior son los agrietamientos y hundimientos del terreno entre 1981 y 1987 y pérdidas del 17"\% de zonas boscosas, principalmente en las partes altas del CARL, a partir del 2000 (Bernardino, 2014; Esteller et al., 2015).

Debe subrayarse que el crecimiento de la mancha urbana de la ZMT ha sido vertiginoso y extensivo, lo que ha ejercido una enorme presión sobre la disponibilidad de agua (Cuadro $\mathrm{N}^{\circ} 2$ ). El proceso de metropolización en Toluca se inicia en la década de los setenta del siglo XX, con la creación de un polo de desarrollo (el corredor industrial Toluca-Lerma), seguido de acelerados procesos migratorios en los ochenta. Actualmente el modelo de crecimiento de la ZMT está determinado por el desarrollo de actividades económicas y del mercado inmobiliario, fundamentalmente expansivo, disperso y desordenado, ocupando grandes superficies territoriales, con baja densidad, dando origen a fenómenos urbanos poco sostenibles, como son la fragmentación y la segregación, generando importantes cambios en la estructura socioespacial de la ciudad (Salgado et al., 2016:193-210; Garrocho et al., 2013).

La delimitación de la "cuenca hidrosocial" de la ZMT (Figura $\mathrm{N}^{\circ} 4$ ) se realizó tomando en consideración las fuentes internas y externas de la dinámica del sistema de abastecimiento de agua, y corresponden al acuífero de Toluca y tres cuencas hidrológicas: importación de agua de la Cuenca del Sistema Cutzamala (CSC), área de captación en el Curso Alto del río Lerma (CARL), así como, la exportación de agua a través del sistema Lerma a la Cuenca del Valle de México $(\mathrm{CVM})^{17}$.

Principalmente a tres municipios del Estado de México (Atizapán de Zaragoza, Naucalpan y Tlalnepantla) y nueve delegaciones de la Ciudad de México (Azcapotzalco, Benito Juárez, Coyoacán, Cuajimalpa, Cuauhtémoc, Magdalena Contreras, Miguel Hidalgo, Iztacalco e Iztapalapa). El sistema Lerma se combina con el sistema Cutzamala para abastecer a la Ciudad de México. 
Cuadro $\mathrm{N}^{\circ} 2$

Expansión de las ciudades en México 1980-2010

\begin{tabular}{|l|r|r|}
\hline Zona Metropolitana & Crecimiento poblacional (\%) & Crecimiento de la mancha urbana (\%) \\
\hline Valle de México & 142 & 357 \\
\hline Guadalajara & 198 & 382 \\
\hline Monterrey & 199 & 490 \\
\hline Puebla-Tlaxcala & 246 & 1.258 \\
\hline Toluca & 341 & 2.690 \\
\hline Tijuana & 356 & 437 \\
\hline
\end{tabular}

Fuente: SEDESOL (2012).

Figura $\mathrm{N}^{\circ} 4$

Delimitación de la "Cuenca Hidrosocial" de la Zona Metropolitana de Toluca

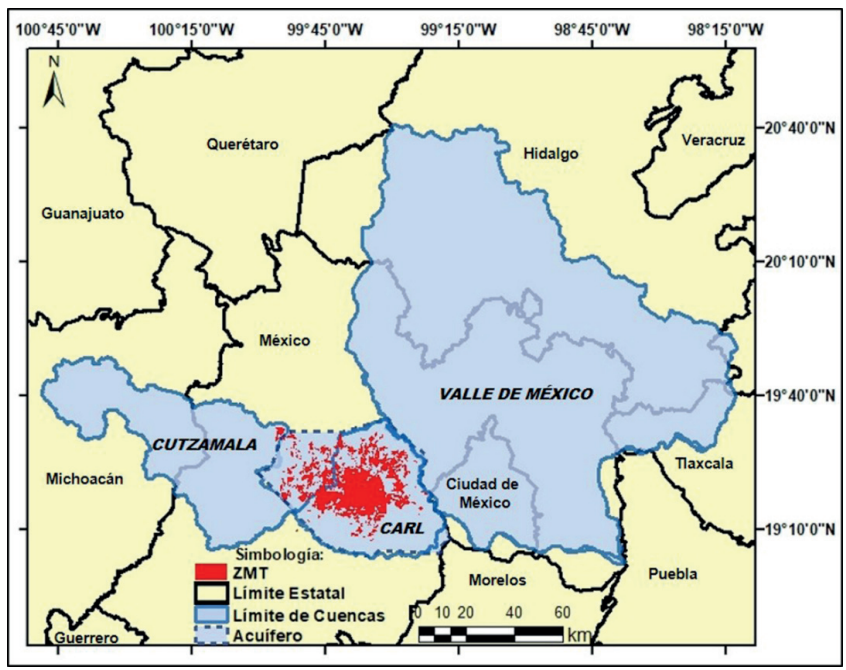

Fuente: Elaboración propia.

\section{Metodología}

El diseño y modelado de escenarios tendenciales del "ciclo hidrosocial urbano" de la ZMT, con base en el esquema metodológico de la modelación de sistemas dinámicos adaptativos, considera la Ecuación de Balance de masas del Sistema Urbano de Abastecimiento de Agua de Martínez y Vargas (2016), que aquí se adapta como se muestra en la ecuación 1.

$$
\frac{d Q}{d t}=\frac{d F}{d t}-\frac{d U}{d t}-\frac{d P}{d t}+\frac{d R}{d t} \quad \text { (Eс. 1) }
$$


Donde:

$Q=$ caudal de ingreso del sistema (que puede ser superávit o déficit).

$F=$ caudal que proporcionan todas las fuentes de abastecimiento (superficiales y subterráneas).

$U=$ caudal de consumo por todos los usos (doméstico, industrial y comercial).

$P=$ caudal que se pierde en el sistema (pérdidas físicas en la red).

$R$ = caudal que se recupera y reingresa al sistema (agua tratada y reusada) más caudales extraordinarios de ingreso (captación de agua pluvial).

Cada una de las variables puede descomponerse en sus respectivos elementos. Por ejemplo: las fuentes de abastecimiento se subdividen en superficiales y subterráneas; los usos en doméstico, comercial e industrial, por lo que la ecuación puede reescribirse como se muestra en la ecuación 2:

$$
\frac{d Q}{d t}=\int_{i=1}^{n} \frac{d F_{i}}{d t}-\int_{i=1}^{n} \frac{d U_{i}}{d t}-\int_{i=1}^{n} \frac{d P_{i}}{d t}+\int_{i=1}^{n} \frac{d R_{i}}{d t}
$$

Donde:

$F_{i}=$ caudal de la $i$-ésima fuente de abastecimiento.

$U_{i}=$ caudal del consumo del $i$-ésimo uso del agua.

$P_{i}=$ caudal que se pierde en el sistema en el $i$-ésimo tipo de pérdida.

$R_{i}=$ caudal que se recupera y reingresa al sistema en el $i$-ésimo método o acción de recuperación.

La mayoría de las $i$-funciones de las variables no son continuas o no pueden integrarse directamente, por lo que es conveniente expresarlas como variables discretas (ecuación 3):

$$
\frac{d Q}{d t}=\sum_{i=1}^{n} \frac{d F_{i}}{d t}-\sum_{i=1}^{n} \frac{d U_{i}}{d t}-\sum_{i=1}^{n} \frac{d P_{i}}{d t}+\sum_{i=1}^{n} \frac{d R_{i}}{d t} \quad \text { (Ec. 3) }
$$

Además, debido a que no pueden integrarse directamente en el tiempo, es necesario considerar la suma con intervalos cercanos de las funciones de estas variables (ecuación 4).

$$
Q^{j+1}=Q^{j}+\sum_{i=1}^{n}\left(F_{i}^{j+1}-F_{i}^{j}\right)-\sum_{i=1}^{n}\left(U_{i}^{j+1}-U_{i}^{j}\right)-\sum_{i=1}^{n}\left(P_{i}^{j+1}-P_{i}^{j}\right)+\sum_{i=1}^{n}\left(R_{i}^{j+1}-R_{i}^{j}\right)
$$

Es fundamental considerar las características sistémicas de interrelación y dependencia de las variables. Por ejemplo, el desarrollo económico de las ciudades favorece el crecimiento demográfico y los procesos de urbanización y metropolización, que generan mayor demanda de agua con el paso del tiempo. Por tanto, en el modelado del "ciclo hidrosocial urbano" de la ZMT se toman en cuenta las siguientes variables y relaciones funcionales:

1. Consumos:

- Consumo doméstico = $\mathrm{f}$ (población, tasa de crecimiento poblacional, dotación, pérdidas físicas).

- Consumo industrial = $f$ (industrias, tasa de crecimiento industrial, dotación para uso industrial, pérdidas físicas).

- Consumo comercial $=\mathrm{f}$ (comercios, tasa de crecimiento comercial, dotación para uso comercial, pérdidas físicas). 
- Consumo áreas verdes $=\mathrm{f}$ (superficie de áreas verdes, coeficiente de riego de jardines, tasa de variación).

- Dotación = f (temperatura, población).

2. Aportaciones:

- Aportaciones $=\mathrm{f}$ (aportaciones subterráneas, aportaciones superficiales adicionales al escurrimiento propio de la cuenca).

- Tasa subterránea $=\mathrm{f}$ (aportaciones de extracción).

3. Aportaciones extraordinarias:

- Pluviales $=f$ (pluvial sin recubrimiento, pluvial con recubrimiento).

- Pluvial con recubrimiento $=\mathrm{f}$ (área de captación cubierta, coeficiente de escurrimiento, precipitación).

- Pluvial sin recubrimiento = f (área de captación abierta, coeficiente de escurrimiento, precipitación).

- Agua residual tratada $=\mathrm{f}$ (aportaciones al sistema, coeficiente de aportación, agua residual tratada).

El modelo permite cuantificar el estado que guarda el balance de masas entre oferta y demanda de agua, derivado de las variables y las interrelaciones que intervienen en el "ciclo hidrosocial urbano" de la ZMT para el periodo 2015-2030. Para ello se utilizó el software Vensim PLE 6.3. Este software de modelado y simulación se fundamenta en técnicas de análisis de dinámica de sistemas bajo un interface gráfico (Diagramas Hidrodinámicos o Diagramas de Forrester), con particular interés en recursos hídricos (Goncalves y Giorgetti, 2013; Abadi et al., 2015; Martínez y Vargas, 2016). ${ }^{18}$

\section{Modelación del "Ciclo Hidrosocial Urbano" de la ZMT}

El modelado y simulación para el periodo 2015-2030 de la oferta y la demanda de agua en la ZMT, corresponde a una representación simplificada, de la compleja interrelación estructural y dinámica del modelo teórico conceptual basado en el sistema agua-sociedad inmersa en la "Cuenca Hidrosocial" (Figura $N^{\circ} 5$ ). Analizado bajo una estructura dispuesta por la asociación por medio de operaciones matemáticas, de factores y variables de presión y disponibilidad de agua, que interactúan entre sí, y que se reconfiguran de manera iterativa en el espacio y en el tiempo, cuya respuesta en su conjunto de la condición de incertidumbre del balance masas entre lo hídrico y lo social, permite comprender y determinar los distintos escenarios, y el estado que guarda el balance hídrico del "ciclo hidrosocial urbano", tendientes al uso sostenible del agua en la ZMT (Figura $\mathrm{N}^{\circ}$ 6). La ponderación de valores de los factores y variables de los procesos naturales y sociales de entrada, que alimentan el modelo, así como también la precisión de las unidades de media y las fuentes de información utilizadas se reportan en el Cuadro $N^{\circ} 3$.

Existen varios programas diseñados para construir modelos lógico-matemáticos de sistemas complejos: Arena, AnyLogic, eLSE, Evolution, iThink/Stella, Matlab/Simulink, Promodel, Powersim, Sigem, Simile, Vensim, por mencionar algunos. Disponible en Internet: http://es.freedownloadmanager.org/Windows-PC/Vensim-PLE2.html 
Figura $\mathrm{N}^{\circ} 5$

Modelo teórico conceptual de simulación

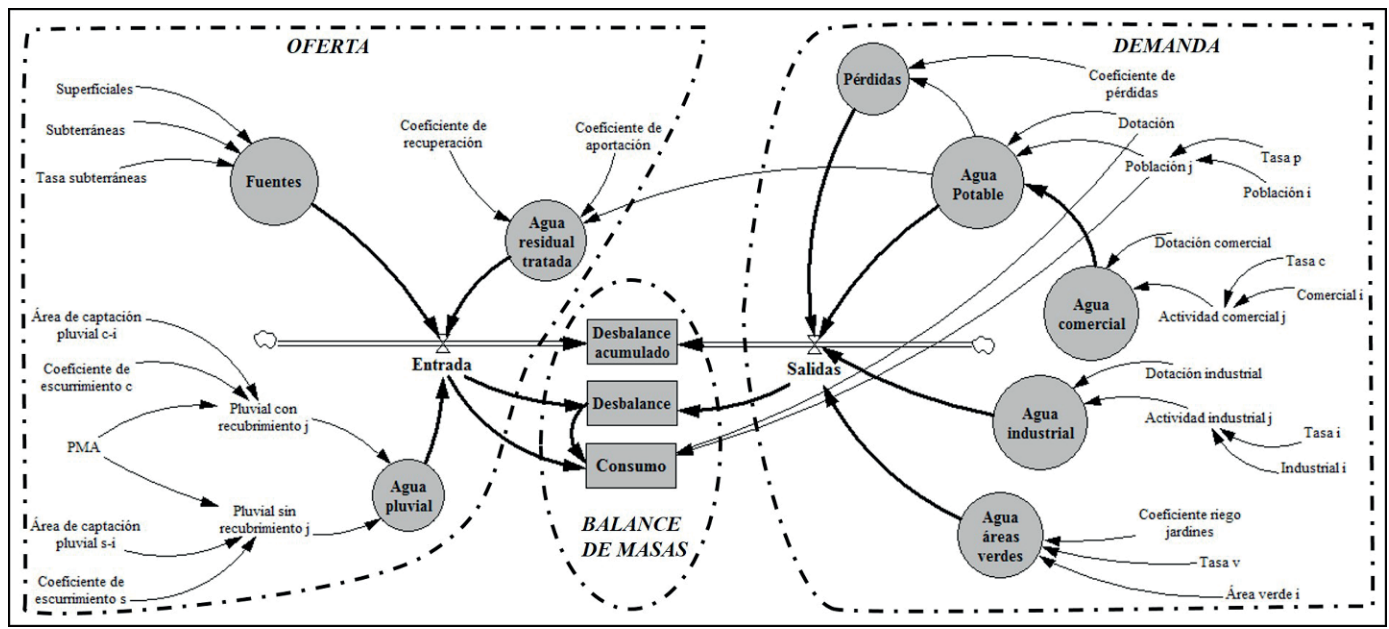

Fuente: Adaptado de Martínez y Vargas (2016).

Figura $\mathrm{N}^{\circ} 6$

Interacción iterativa de factores y variables del modelo

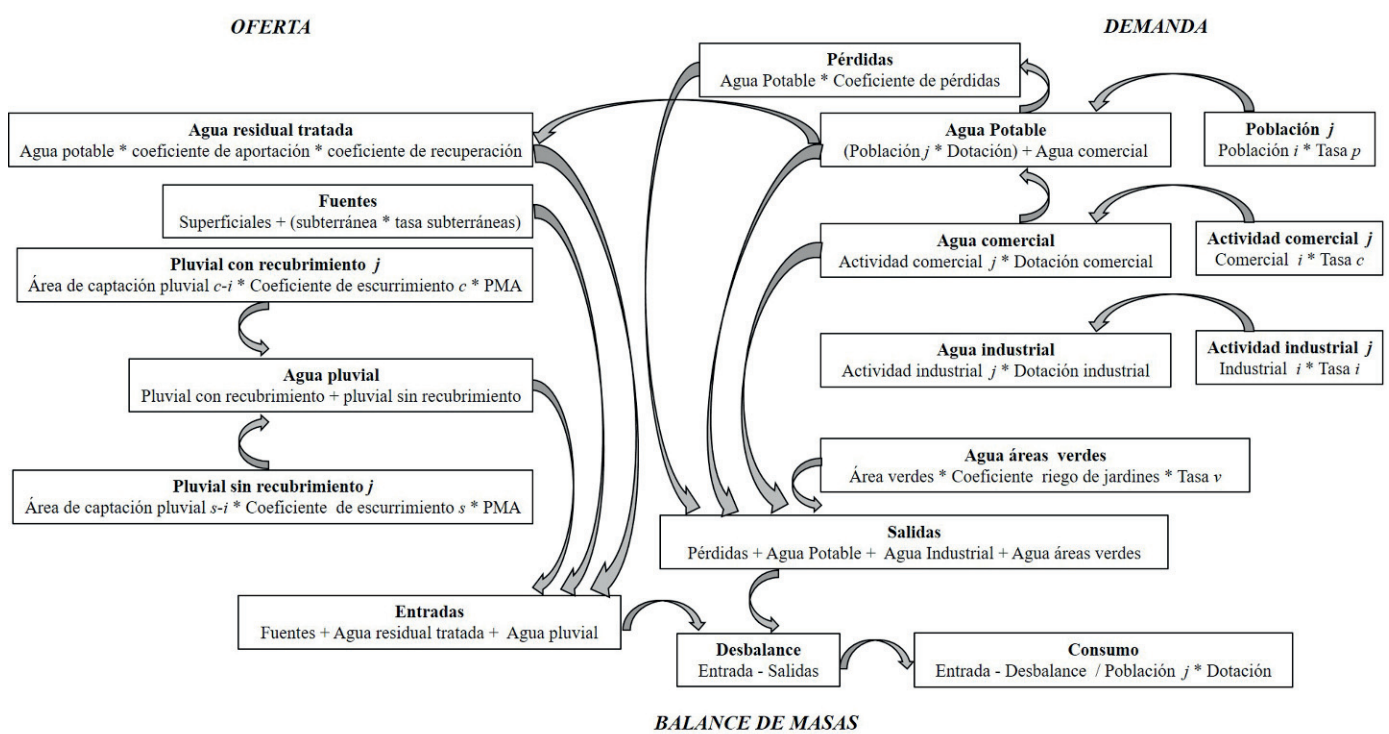

Fuente: Elaboración propia.

Nos enfocamos a analizar tres escenarios: $i$. Continuar bajo las mismas condiciones y presiones actuales, excepto por el acelerado proceso de crecimiento de la ZMT; ii. Prescindir del abasto del Sistema Cutzamala; iii. Limitar la exportación de agua al Valle de México. La selección de los escenarios se hizo a partir de la viabilidad y tendencias observadas en la codependencia territorial 
de la "cuenca hidrosocial", así como de la dinámica de los caudales entre las fuentes de abastecimiento (oferta) y el crecimiento poblacional (demanda) (Figura $\left.N^{\circ} 7\right)^{19}$.

\section{Cuadro $\mathrm{N}^{\circ} 3$}

Variables y datos utilizados para la simulación del modelo dinámico adaptativo de la ZMT

\begin{tabular}{|c|c|c|c|}
\hline Variable & Unidades & Descripción y fuente de información & $\begin{array}{c}\text { Valor en el } \\
\text { modelo }\end{array}$ \\
\hline $\begin{array}{l}\text { Coeficiente de } \\
\text { pérdidas }\end{array}$ & Adimensional & $\begin{array}{l}\text { En los sistemas de abastecimiento de agua } \\
\text { potable, las pérdidas físicas son de aproxima- } \\
\text { damente del } 40 \text { por ciento (MAPAS-CONA- } \\
\text { GUA, 2015). }\end{array}$ & $40 \%$ \\
\hline Dotación & I/hab/d & $\begin{array}{l}\text { Dotación estimada a partir de valores de ex- } \\
\text { tracción del acuifero y datos del Programa de } \\
\text { Indicadores de Gestión de Organismos Ope- } \\
\text { radores (PIGOO, 2015). }\end{array}$ & 210,0 \\
\hline $\begin{array}{c}\text { Tasa } p \\
\text { (tasa de crecimiento } \\
\text { poblacional) }\end{array}$ & Adimensional & $\begin{array}{l}\text { Tasa de crecimiento medio anual con base } \\
\text { en los Censos de Población y Vivienda } 1990 \text {, } \\
2000 \text { y } 2010 \text { del INEGI y las proyecciones de la } \\
\text { población } 2010-2050 \text { de la CONAPO. }\end{array}$ & $1,5 \%$ \\
\hline Población $i$ & Habitantes & $\begin{array}{l}\text { Población asentada en la ZMT para el año 2015, } \\
\text { con base en la Encuesta Intercensal } 2015 .\end{array}$ & 2.116 .506 \\
\hline Población $j$ & Habitantes & Población de proyecto (horizonte 2030). & 2.646 .124 \\
\hline $\begin{array}{c}\text { Tasa } i \\
\text { (tasa de crecimiento } \\
\text { industrial) } \\
\end{array}$ & Adimensional & $\begin{array}{l}\text { Tasa media anual considerada con base en el } \\
\text { Directorio Estadístico Nacional de Unidades } \\
\text { Económicas } 2010 \text { del INEGI. }\end{array}$ & $2,6 \%$ \\
\hline Industrial $i$ & Adimensional & $\begin{array}{l}\text { Dato de industrias manufactureras asentadas } \\
\text { en la ZMT, con base en el Directorio Estadís- } \\
\text { tico Nacional de Unidades Económicas } 2010 \\
\text { del INEGI. }\end{array}$ & 9.661 \\
\hline Actividad industrial $j$ & Adimensional & Industrias de proyecto (horizonte 2030). & 14.198 \\
\hline $\begin{array}{c}\text { Tasa c } \\
\text { (tasa de crecimiento } \\
\text { comercial) }\end{array}$ & Adimensional & $\begin{array}{l}\text { Tasa media anual considerada con base en el } \\
\text { Directorio Estadístico Nacional de Unidades } \\
\text { Económicas } 2010 \text { del INEGI. }\end{array}$ & $2,3 \%$ \\
\hline Comercial $i$ & Adimensional & $\begin{array}{l}\text { Dato de comercios asentados en la ZMT, con } \\
\text { base en el Directorio Estadístico Nacional de } \\
\text { Unidades Económicas } 2010 \text { del INEGI. }\end{array}$ & 38.907 \\
\hline $\begin{array}{c}\text { Actividad comercial } \\
j\end{array}$ & Adimensional & Comercios de proyecto (horizonte 2030). & 54.722 \\
\hline
\end{tabular}

19 Se construyeron múltiples escenarios, pero estos son de los más ilustrativos de la potencia de nuestro modelo. 
Continuación Cuadro $N^{\circ} 3$

\begin{tabular}{|c|c|c|c|c|}
\hline & Variable & Unidades & Descripción y fuente de información & $\begin{array}{l}\text { Valor en el } \\
\text { modelo }\end{array}$ \\
\hline & $\begin{array}{c}\text { Tasa } v \\
\text { (tasa de áreas } \\
\text { verdes) }\end{array}$ & Adimensional & $\begin{array}{l}\text { Porcentaje estimado de áreas verdes urbanas, } \\
\text { considerado en los Planes Municipales de De- } \\
\text { sarrollo. }\end{array}$ & $0,2 \%$ \\
\hline & Área verde $i$ & $\mathrm{Ha}$ & $\begin{array}{l}\text { Superficie estimada de parques y jardines, } \\
\text { con base en el Inventario de áreas verdes mu- } \\
\text { nicipales y Planes Municipales de Desarrollo. }\end{array}$ & 692 \\
\hline & $\begin{array}{l}\text { Coeficiente de riego } \\
\text { en jardines }\end{array}$ & $\mathrm{l} / \mathrm{m}^{2} / \mathrm{d}$ & $\begin{array}{l}\text { Datos medios diarios necesarios para riego } \\
\text { de jardines, con base en el Manual para el uso } \\
\text { eficiente y racional del agua (Bourguett et al, } \\
\text { 2003). }\end{array}$ & 7,2 \\
\hline & $\begin{array}{l}\text { Coeficiente de } \\
\text { pérdidas }\end{array}$ & Adimensional & $\begin{array}{l}\text { En zonas habitacionales, se adopta como } \\
\text { aportación de aguas residuales el } 75 \% \text { de la } \\
\text { dotación de agua potable (MAPAS-CONAGUA, } \\
2015 \text { ). }\end{array}$ & $75 \%$ \\
\hline & $\begin{array}{l}\text { Coeficiente de } \\
\text { recuperación }\end{array}$ & Adimensional & $\begin{array}{l}\text { Actualmente no se realiza aprovechamiento } \\
\text { de agua residual tratada para reutilizarla. }\end{array}$ & 0 \\
\hline$\frac{\pi}{0}$ & $\begin{array}{l}\text { Superficiales } \\
\text { (adicionales al } \\
\text { escurrimiento } \\
\text { propio de la } \\
\text { cuenca) }\end{array}$ & $\mathrm{m}^{3} / \mathrm{s}$ & $\begin{array}{l}\text { La ZMT se abastece con } 22.20 \mathrm{Hm}^{3} \text { /año de la } \\
\text { Presa Miguel Alemán (Valle de Bravo) a través } \\
\text { del Sistema Cutzamala (Korenfeld, 2009). }\end{array}$ & 0,704 \\
\hline 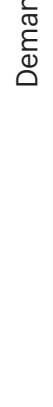 & Subterráneas & $\mathrm{m}^{3} / \mathrm{s}$ & $\begin{array}{l}\text { El Acuífero de Toluca de acuerdo con la Ac- } \\
\text { tualización de la Disponibilidad Media Anual } \\
\text { de Agua Subterránea, publicado en el Diario } \\
\text { Oficial de la Federación (DOF } 20 \text { de abril 2015) } \\
\text { reporta una extracción de } 422.4 \mathrm{Hm}^{3} / \text { año. Pre- } \\
\text { cisar que de esta extracción } 160.9 \mathrm{Hm}^{3} / \text { año, } \\
\text { son enviados a la Zona Metropolitana del Valle } \\
\text { de México a través del sistema Lerma (CONA- } \\
\text { GUA-SACMEX). }\end{array}$ & 8,29 \\
\hline & Tasa Subterránea & $\mathrm{Hm}^{3} / a n ̃ o$ & 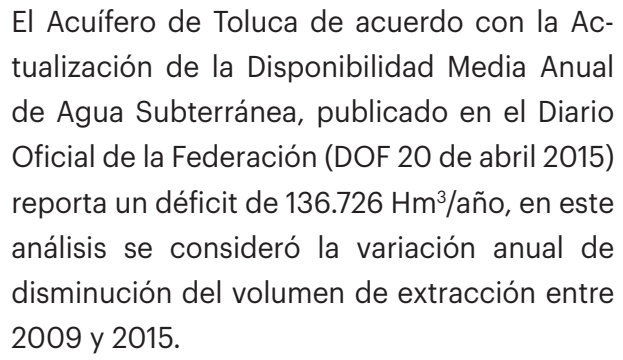 & $-2,9$ \\
\hline
\end{tabular}


Continuación Cuadro $N^{\circ} 3$

\begin{tabular}{|c|c|c|c|c|}
\hline & Variable & Unidades & Descripción y fuente de información & $\begin{array}{c}\text { Valor en el } \\
\text { modelo }\end{array}$ \\
\hline \multirow{5}{*}{$\begin{array}{l}\frac{\pi}{0} \\
\frac{C}{\sigma} \\
\frac{\sigma}{0} \\
0 \\
0\end{array}$} & $\begin{array}{l}\text { Área de captación } \\
\text { pluvial c-i } \\
\text { (de áreas cubiertas) }\end{array}$ & ha & $\begin{array}{l}\text { Al no existir infraestructura de captación, se } \\
\text { hizo el supuesto de superficie de áreas verdes, } \\
\text { tomando en consideración la relación anual } \\
\text { de captación de } 1 \mathrm{l} / \mathrm{m}^{2} \text {, en función de la preci- } \\
\text { pitación media anual y materiales de cubierta } \\
\text { para su captación. }\end{array}$ & 692 \\
\hline & $\begin{array}{l}\text { Coeficiente de } \\
\text { escurrimiento c }\end{array}$ & Adimensional & $\begin{array}{l}\text { Coeficiente de escurrimiento para superficies } \\
\text { con recubrimiento calculado con base en la } \\
\text { NOM-011-CONAGUA-2015. }\end{array}$ & 0,340 \\
\hline & PMA & $\mathrm{mm}$ & $\begin{array}{l}\text { Valor estimado de precipitación media anual } \\
\text { considerando datos de estaciones climatoló- } \\
\text { gicas de la ZMT. }\end{array}$ & 900,0 \\
\hline & $\begin{array}{l}\text { Área de captación } \\
\text { pluvial s-i } \\
\text { (de áreas no } \\
\text { cubiertas) }\end{array}$ & ha & $\begin{array}{l}\text { Se consideran como áreas potenciales, las } \\
\text { superficies estimadas de parques y jardines, } \\
\text { con base en el Inventario de áreas verdes mu- } \\
\text { nicipales y Planes Municipales de Desarrollo. } \\
\text { Tomando en consideración la relación anual } \\
\text { de captación de } 1 \mathrm{l} / \mathrm{m}^{2} \text {, en función de la preci- } \\
\text { pitación media anual y materiales de cubierta } \\
\text { para su captación. }\end{array}$ & 692 \\
\hline & $\begin{array}{l}\text { Coeficiente de } \\
\text { escurrimiento } s\end{array}$ & Adimensional & $\begin{array}{l}\text { Coeficiente de escurrimiento para superficies } \\
\text { con cubierta vegetal calculado con base en la } \\
\text { NOM-011-CONAGUA-2015. }\end{array}$ & 0,109 \\
\hline
\end{tabular}

Adicionalmente a estos escenarios, se señalan tres acciones emergentes a nivel local, consideradas factibles y de alto impacto a las presiones tendenciales: a. Reutilización de agua residual; b. Reducción de pérdidas físicas en infraestructura; y, c. Aprovechamiento de agua de lluvia.

\section{Escenarios}

Escenario 1. Se mantienen las condiciones actuales, salvo el crecimiento poblacional de la ZMT. De acuerdo con la proyección del crecimiento poblacional (que eleva la demanda de agua), se pasaría de 2,1 millones de habitantes en 2015, a 2,6 millones en 2030. Esto, aunado al decremento de la oferta por reducción de caudales de las fuentes de abastecimiento de agua, genera un desbalance del sistema, representado por una reducción del 42,0\% en la dotación de agua per cápita, pasando de 210,0 I/hab/d en 2015, a 120,0 I/hab/d en 2030. Lo que implica 75,0 I/hab/d, por debajo de los valores de diseño para dotación de agua para uso doméstico de la CONAGUA de 195,0 l/hab/d en un nivel socioeconómico medio y clima templado. Cabe señalar que este escenario también implica el requerimiento del desarrollo tecnológico y su implementación que haga posible esta reducción en la dotación per cápita. 


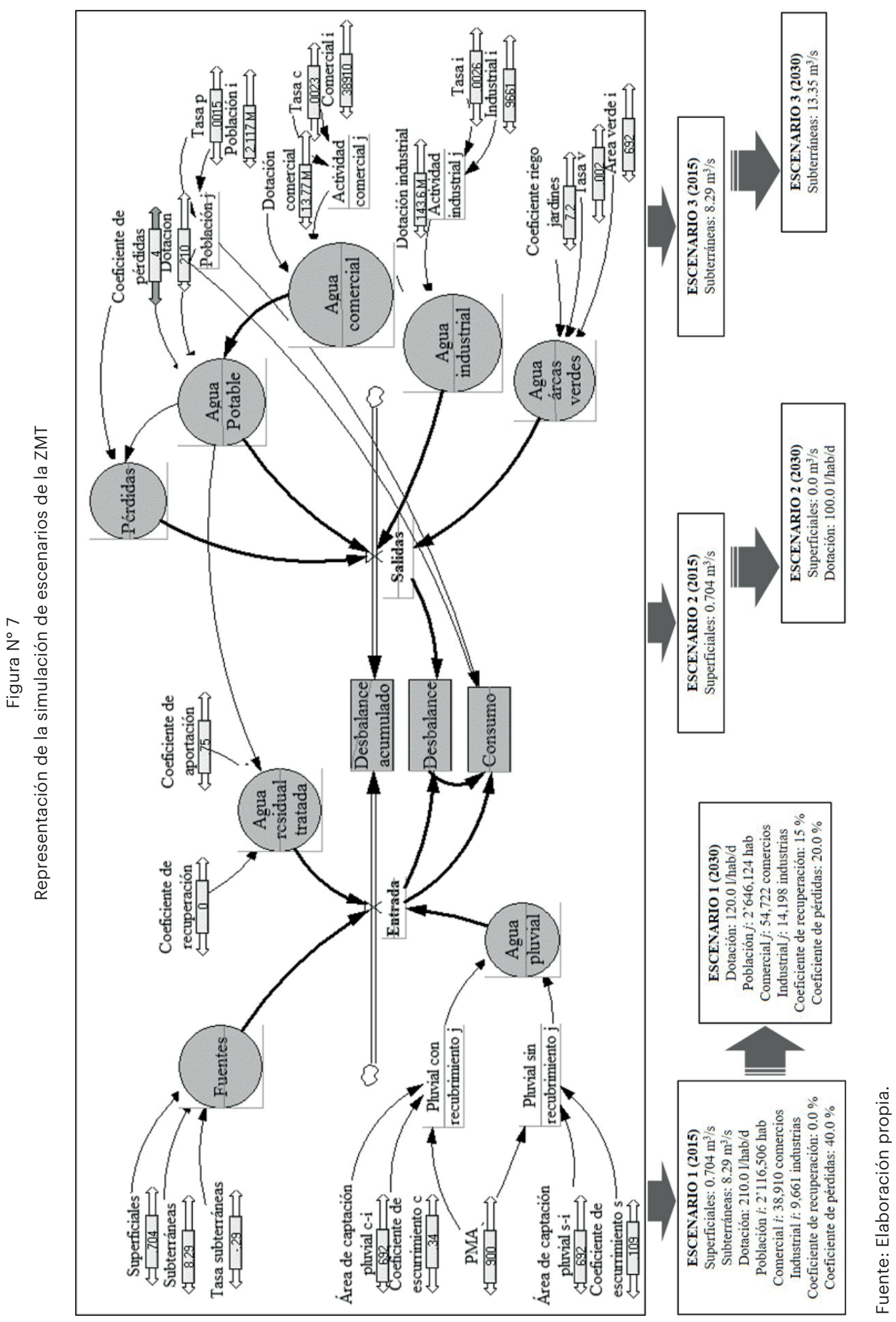


Escenario 2. Prescindir del abastecimiento del Sistema Cutzamala. Se reconoce en el modelo que la permanencia y eficiencia de este sistema no están garantizadas, debido a diversos factores técnicos y conflictos históricos de carácter ambiental, social y territorial, que son crecientes por la falta de coordinación institucional y de gobernabilidad hídrica (Banco Mundial-CONAGUA, 2015). ${ }^{20}$ Por lo anterior, el modelo considera un periodo de vida útil de la infraestructura y capacidad de abastecimiento del sistema de sólo 10 años. El modelo indica que terminada la vida útil del sistema se tendría un déficit de $52.5 \%$ en la dotación per cápita de la ZMT. Es decir: una reducción de 210,0 l/hab/d en 2015 a 100,0 l/hab/d en 2030. Esta situación resultaría catastrófica, por lo que es urgente instrumentar acciones de alto impacto como parte de un plan integral de largo plazo. Dado que no existen otras fuentes emergentes de abastecimiento para la ciudad, la ZMT es altamente vulnerable al declive del sistema Cutzamala.

Escenario 3. No se exporta agua al Valle de México. Esta consideración obedece a que al término de 2015 venció el título de concesión núm. 5DFE100309/26HMSG96, otorgado en 1996, para la extracción y trasvase anual de un caudal de $149,7 \mathrm{Hm}^{3}$ de los acuíferos de Toluca e Ixtlahuaca-Atlacomulco, al Valle de México. Resaltar que el modelo estima que bajo este esquema no se logra alcanzar un equilibrio del balance oferta-demanda de agua en el acuífero de Toluca, sin embargo, lo más recomendable es que este volumen equivalente a $93,0 \mathrm{Hm}^{3}$, fuera considerado como almacenamiento para mitigar la sobreexplotación del acuífero, situación que sería favorable siempre y cuando se limitara la extracción de agua del acuífero, al contar con un mayor caudal de recuperación, tendiente al equilibrio y disminución del estrés hídrico.

Se debe señalar que este escenario resulta poco viable en términos sociales y políticos. La presión y demanda de agua de los municipios beneficiados del Estado de México y de las delegaciones de la Ciudad de México, es muy alta. No obstante, este escenario abre un área de oportunidad y sugiere una serie de cuestionamientos y líneas de renegociación que deben ser abordados a la brevedad entre los gobiernos del Estado de México y la Ciudad de México.

\section{Acciones emergentes}

Acción emergente 1. Reutilización de agua residual. El supuesto es incorporar al sistema de abastecimiento de agua la reutilización gradual de agua residual urbana, lo que permitiría incrementar el volumen del caudal tratado y garantizar la calidad del agua bajo la NOM-003-SEMARNAT-199721. En el modelo se consideró un incremento de $15 \%$ del volumen tratado, con un esperado de $36,38 \mathrm{Hm}^{3}$ en el año 2030, equivalente a la demanda de aproximadamente 470 mil habitantes. Esta situación puede incrementarse en volumen y disminuirse en el tiempo de su incorporación, en función de la inversión y mejora de la infraestructura de tratamiento. Especialmente de las Macroplantas Toluca Norte y Toluca Oriente, las plantas municipales, así como de Reciclagua Ambiental, en el caso de aguas residuales de tipo industrial.

Acción emergente 2. Reducción de pérdidas físicas en infraestructura. Se supone que las pérdidas físicas de agua en la infraestructura de conducción y distribución se reducen $50 \%$. Para esto

\footnotetext{
20 Ver el Diagnóstico del Sistema Cutzamala. Disponible en Internet: https://www.gob.mx/cms/uploads/attachment/file/110933/Cutzamala_ Diagnostico_integral_parte_1.pdf

${ }_{21}$ Norma Oficial Mexicana que establece los limites máximos permisibles de contaminantes para aguas residuales tratadas que se reutilicen en servicios al público.
} 


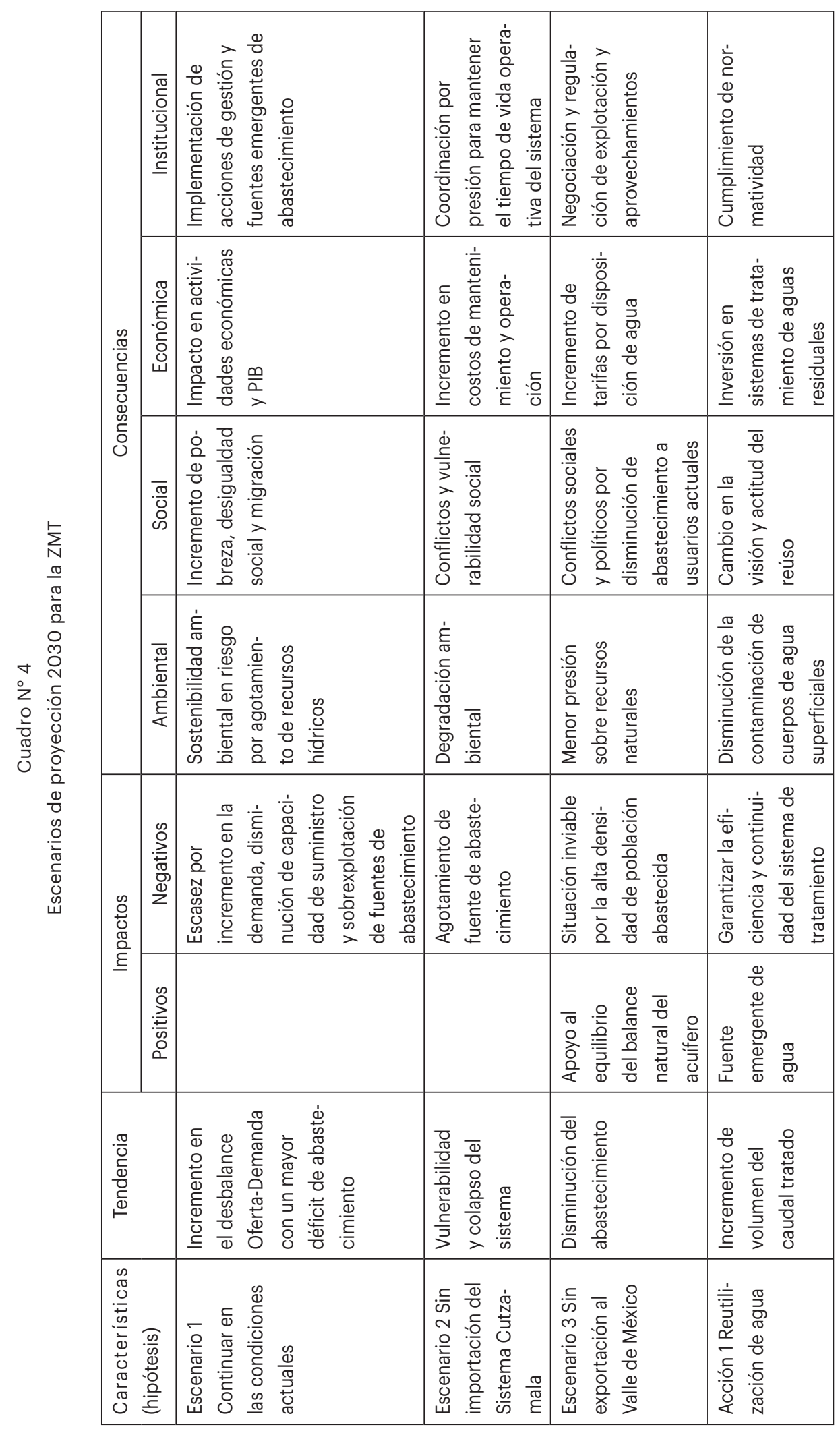




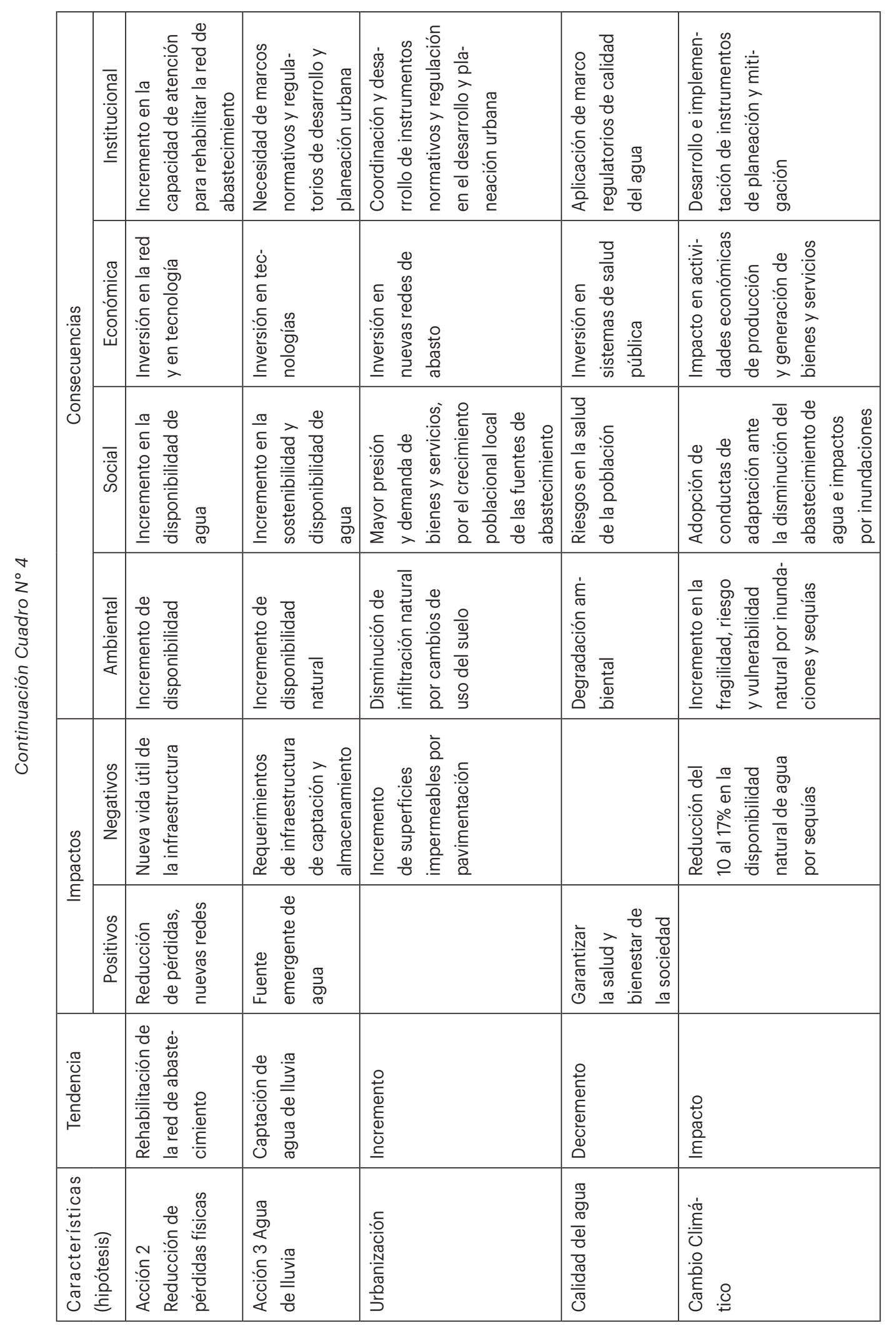

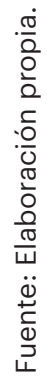


se requerirían trabajos de mantenimiento y rehabilitación en un esquema de mejora continua de la red de distribución, actualmente realizados por organismos operadores de agua y saneamiento en puntos específicos. La red no ha sido atendida con la intensidad suficiente durante los últimos 45 años. Esta acción podría reportar un incremento en la disponibilidad de $24,3 \mathrm{Hm}^{3}$ en el año 2030, equivalente a la demanda de aproximadamente 320 mil habitantes.

Acción emergente 3. Aprovechamiento de agua de lluvia. El supuesto es establecer marcos normativos y regulatorios que permitan implantar alternativas sostenibles de abastecimiento de agua en los nuevos esquemas de vivienda del sector inmobiliario. La idea sería que las superficies de aprovechamiento cubrieran las necesidades anuales de agua de una familia. Esto reduciría notablemente extracciones adicionales a las fuentes de abastecimiento. Bajo este supuesto y de acuerdo con las condiciones climáticas de la ZMT y a su precipitación media anual de $900 \mathrm{~mm}$, sería posible captar $900 \mathrm{l} / \mathrm{año} / \mathrm{m}^{2}$ durante los meses de junio a septiembre si se dispusiera de la infraestructura necesaria de captación y almacenamiento.

El Cuadro $\mathrm{N}^{\circ} 4$ resume los impactos (positivos y negativos) y consecuencias (en las dimensiones ambiental, social, económica e institucional) de los escenarios de proyección 2030 y acciones emergentes factibles. También se presentan manera enunciativa algunas variables no consideradas en el modelo y que, de incluirse, permitirían informar mejor la toma de decisiones.

\section{Conclusiones}

El modelo conceptual del ciclo hidrológico urbano de la ZMT se encuentra representado por la gestión territorial definida en su "cuenca hidrosocial". Bajo este enfoque es posible considerar las complejas externalidades de dependencia en importaciones de agua del Sistema Cutzamala, exportaciones al Valle de México, así como las internalidades superficiales del CARL y las subterráneas del acuífero de Toluca, en el contexto del acelerado crecimiento poblacional y desarrollo económico de la ciudad. En el futuro próximo todos estos vectores tendrán impactos y consecuencias "sociohídricas" altamente significativas.

Las estimaciones del modelo en materia de disponibilidad de agua para 2030 indican que la sostenibilidad de la ZMT está en riesgo. El balance hídrico del "ciclo hidrosocial urbano" y el patrón del abastecimiento de agua, registran un déficit en el balance oferta-demanda que se irá acentuando, dado el incremento continuo de la demanda y el declive de la oferta. La ZMT registra una alta vulnerabilidad social, tanto en la disponibilidad de agua como en la baja resiliencia urbana, que se manifiesta en la poca capacidad del sistema-ciudad para absorber cambios y perturbaciones sociohídricas. Resulta clave reconocer el importante rol de las variables críticas emergentes, de atención y disminución de fugas físicas en la red de abastecimiento, la disponibilidad y la capacidad de reutilizar agua residual tratada, así como la captación de agua de lluvia. Esta última acción debería ser considerada en la normatividad para el ordenamiento de nuevos desarrollos habitacionales en la ZMT.

Para lograr eficiencia en la gobernanza y gobernabilidad del "ciclo hidrosocial urbano" de la ZMT, se requiere de una gestión integrada del agua como soporte de carácter científico-técnico para alimentar las políticas públicas. Es precisamente ahí, donde radica la potencialidad de apli- 
cación del presente trabajo, cuyos resultados ofrecen áreas de oportunidad, indicando la urgente necesidad de implementar en el menor tiempo posible, marcos jurídicos regulatorios y normativos que salvaguarden la supervivencia, desarrollo y sostenibilidad de la ZMT e incrementen su resiliencia, siempre y cuando se caractericen por un entorno político con objetivos y metas comunes, sin conflicto de interés entre los distintos actores y disminución de los niveles de corrupción.

Finalmente se abre un amplio panorama de posibilidades para abordar el análisis y el estudio de las ciudades, que, por sus cualidades, es capaz de ser replicado y aplicado a diversos espacios urbanos. La prosperidad o colapso de la sociedad, dependerá de su capacidad de respuesta, adaptación y prevención del riesgo por escasez de agua a que están expuestas las ciudades.

\section{Aportaciones}

Se señalan aspectos relevantes que son aportados por este trabajo:

i. Base teórico-conceptual de perspectiva crítica para el análisis y comprensión del ciclo hidrosocial urbano;

ii. Adopción de una dimensión espacial socioterritorial de análisis correspondiente a la cuenca hidrosocial y su implicación en la evaluación de la dimensión social del problema de escasez de agua;

iii. Posicionamiento estratégico del agua, como factor crítico para lograr el desarrollo sostenible, incrementar la resiliencia y disminuir la vulnerabilidad social;

iv. Herramienta para la toma de decisiones, articulación de sinergias y competencias administrativas, dentro de los esquemas de gestión del agua y planeación de zonas metropolitanas, favoreciendo anticipar impactos y mitigar consecuencias.

\section{Agenda de investigación}

Los siguientes serían los temas prioritarios para avanzar en la construcción de un mejor modelo del "ciclo hidrosocial urbano" de la Zona Metropolitana de Toluca (ZMT):

i. Evaluar el costo económico del abastecimiento de agua, los esquemas tarifarios y costos energéticos de producción, distribución y bombeo, así como el esquema de valoración del recurso agua. Esto repercute en el pago realizado por el consumidor, generando recursos para financiar la infraestructura requerida;

ii. Realizar un análisis espacial a mayor detalle (nivel municipal), a fin de conocer la desigualdad espacial de la distribución de agua y las áreas afectadas por hundimientos y agrietamientos del terreno (por efectos de la variabilidad espacial de la extracción del acuífero), considerando parámetros de calidad del agua, aspecto prioritario en materia de salud de la población.

\section{Referencias bibliográficas}

ABADI, L.S.K.; SHAMSAI, A. \& GOHARNEJAD, H. An analysis of the sustainability of basin water resources using Vensim model. KSCE Journal of Civil Engineering, 2015, Vol. 19, № 6, p. 1941-1949. 
AGUILERA KLINK, F. Hacia una nueva economía del agua: cuestiones fundamentales. Polis. Revista Latinoamericana, 2006, № 14. Disponible en Internet: http://polis.revues.org/5044

ARAGÓN, J.H. y CÓRDOVA, J.J.B. La economía del agua: una aproximación para su gestión responsable. Aventuras del pensamiento. Synthesis, 2009, N 49, p. 1-7.

BAKKER, K. Neoliberalizing nature? Market environmentalism in water supply in England and Wales. Annals of the association of American Geographers, 2005, Vol. 95, Nº 3, p. 542-565.

BANISTER, J. M. Are you Wittfogel or against him? Geophilosophy, hydro-sociality, and the state. Geoforum, 2014, Vol. 57, p. 205-214.

BATTY, M. The new science of cities. Cambridge: MIT Press, 2013.

BERNARDINO, L. Sistema Lerma: una visión política en la gestión pública del agua, ¿solución Estatal o Federal? Toluca: Instituto de Administración Pública del Estado de México, A.C., 2014. ISBN: 978-607-8087-16-7.

BROTO, V.C.; ALLEN, A. \& RAPOPORT, E. Interdisciplinary perspectives on urban metabolism. Journal of Industrial Ecology, 2012, Vol. 16, N 6, p. 851-861.

BOURGUETT, V.J.; CASADOS, J.; MIRELES, V.; GONZÁLEZ, E.; HANSEN, M.; BUENFIL, M. \& CERVANTES, M. Manual para el uso eficiente y racional del agua. iUtiliza sólo la necesaria! Jiutepec: Instituto Mexicano de Tecnología del agua, 2003.

BUDDS, J. Whose scarcity? The hydrosocial cycle and the changing waterscape of La Ligua river basin. In: GOODMAN, M. K., BOYKOFF, M., \& EVERED, K. (editors). Contentious geographies: Environment, meaning, scale. Aldershot: Ashgate, 2008, p. 59-68.

BUDDS, J. Contested H2O: science, policy and politics in water resources management in Chile. Geoforum, 2009, Vol. 40, N³, p. 418-430.

BUDDS, J. La demanda, evaluación y asignación del agua en el contexto de escasez: un análisis del ciclo hidrosocial del valle del río La Ligua, Chile. Revista de Geografía Norte Grande, 2012, Vol. 52, No 1, p. 167-184.

BUDDS, J. \& HINOJOSA, L. Restructuring and rescaling water governance in mining contexts: The co-production of waterscapes in Peru. Water Alternatives, 2012, Vol. 5, No 1, p. 119.

CARDONA ARBOLEDA, O. D. Estimación holística del riesgo sísmico utilizando sistemas dinámicos complejos. Barcelona: Tesis Doctoral, Escola Técnica Superior D’Enginyers de Camins, Canals I Ports, Universitat Politécnica de Cataluya, 2001.

CASTILLO-VILLANUEVA, L. y VELÁZQUEZ-TORRES, D. Sistemas complejos adaptativos, sistemas socio-ecológicos y resiliencia. Quivera, 2015, Vol. 17, № 2, p. 11-32. 
CENTRO AGUA. Proyecto GIRH: Construcción de una Estrategia para promover la Gestión Integral de Recursos Hídricos Cuenca Hidrosocial Pucara. La Paz: Centro Andino para la Gestión y Uso de Agua. 2009. Disponible en internet: http://www.centro-agua.org/proyecto-girh/

CERVANTES-JIMÉNEZ, M.; MASTACHI-LOZA, C. A.; DÍAZ-DELGADO, C.; GÓMEZ-ALBORES, M. Á. \& GONZÁLEZ-SOSA, E. Socio-Ecological Regionalization of the Urban Sub-Basins in Mexico. Water, 2017, Vol. 9, No 1, p. 14.

COMISIÓN NACIONAL DEL AGUA (CONAGUA). Manual de Agua Potable Alcantarillado y Saneamiento (MAPAS). México: Comisión Nacional del Agua, 2015. Disponible en internet: http://aneas. com.mx/manual-de-agua-potable-alcantarillado-y-saneamiento-mapas-conagua-2015/

CONAPO. Proyecciones de la Población de México 2005-2050. México: Consejo Nacional de Población, 2011.

CONSTAS, M.; FRANKENBERGER, T. \& HODDINOTT, J. Resilience measurement principles: Toward an agenda for measurement design. Food Security Information Network, Resilience Measurement Technical Working Group, Technical Series, 2014, № 1. Disponible en Internet: http://www.fao.org/ fileadmin/user_upload/drought/docs/FSIN\%20Resilience\%20Measurement\%20201401.pdf

CRUZ, A.C.P. y MARTÍNEZ, P.C.C. El recurso agua en el entorno de las ciudades sustentables. CULCYT, 2009, Vol. 6, No 31, p. 15.

DAMONTE-VALENCIA, G.H. Redefiniendo territorios hidrosociales: control hídrico en el Valle de Ica, Perú (1993-2013), Cuadernos de Desarrollo Rural, 2015, Vol. 12, № 76, p. 109-133.

DOMíNGUEZ, J. Hacia una buena gobernanza para la gestión integrada de los recursos hídricos. Documento temático de las Américas. Marsella: VI Foro Mundial del Agua, 2012.

DIAMOND, J. Guns, germs, and steel: The fates of human societies. New York: W.W. Norton, 1997.

DIAMOND, J. Colapso: Por qué unas sociedades perduran y otras desaparecen. Barcelona: Debate, Random House Mondadori, 2007.

DÍAZ-DELGADO, C.; FONSECA, C.R.; ESTELLER, M.V.; GUERRA-COBIÁN, V.H., \& FALL, C. The establishment of integrated water resources management based on emergy accounting. Ecological Engineering, 2014, Vol. 63, p. 72-87.

DURÁN, A. y DELGADILLO, O. Conceptos clave para encarar la gestión integral de recursos hídricos en la cuenca hidrosocial Pucara. La Paz: Centro AGUA, documento de trabajo, 2009.

ESTELLER, M.V. \& DIAZ-DELGADO, C. Environmental effects of aquifer overexploitation: a case study in the Highlands of Mexico. Environmental Management, 2002, Vol. 29, N² 2, p. 266-278.

ESTELLER, M.V., EXPÓSITO CASTILLO, J.L.; DÍAZ-DELGADO, C.; PAREDES, J. \& FONSECA, C.R. EXplotación intensiva del acuífero del Valle de Toluca: análisis de algunos efectos económicos-am- 
bientales. En: Fall, C. (coordinador). Avances en Ciencias del Agua. México: Universidad Autónoma del Estado de México, 2015, p 15-31.

FICOT, D. Complexity Theory and Emergence of Water Recycling. London: MSc Thesis, UCLDepartment of Civil, Environmental and Geomatic Engineering, University College London, 2009.

GARROCHO, C. Dinámica de las ciudades de México en el siglo XXI: cinco vectores clave para el desarrollo sostenible. Zinacantepec: ONU, Fondo de Población-CONAPO-El Colegio Mexiquense, 2013.

GARROCHO, C.; ÁLVAREZ-LOBATO, JA. \& CHÁVEZ, T. Calculating intraurban agglomeration of economic units with planar and network K-functions: a comparative analysis. Urban Geography, 2013, Vol. 34, $N^{\circ}$ 2, p. 261-286.

GIACOMONI, M. H.; KANTA, L. \& ZECHMAN, E. M. Complex adaptive systems approach to simulate the sustainability of water resources and urbanization. Journal of Water Resources Planning and Management, 2013, Vol. 139, № 5, p. 554-564.

GONCALVES, J.C.S.I. \& GIORGETTI, M. F. Mathematical Model for the Simulation of Water Quality in Rivers using the Vesnim PLE ${ }^{\circledR}$ Software. Journal of Urban and Environmental Engineering (JUEE), 2013, Vol. 7, No 1, p. 48-63.

GRIMM, N. B.; GROVE J.M., PICKETT, S.T.A. \& REDMAN, C.L. Integrated Approaches to Long-Term Studies of Urban Ecological Systems: Urban ecological systems present multiple challenges to ecologists-pervasive human impact and extreme heterogeneity of cities, and the need to integrate social and ecological approaches, concepts, and theory. BioScience, 2000, Vol. 50, N 7, p. 571-584.

GUTIÉRREZ HERNÁNDEZ, J.E. y MADEREY RASCÓN, L. E. Los Problemas del agua y de las cuencas hidrológicas vinculados a las ciudades. Estudio de casos de las Repúblicas de México y Cuba. México: Facultad de Geografía de la Universidad de La Habana-Instituto de Geografía de la Universidad Nacional Autónoma de México, 2013.

GLOBAL WATER PARTHNERSHIP (GWP). Aumentar la seguridad hídrica. Suecia: 2013.

HASSING, J.; IPSEN, N.; CLAUSEN, T.J.; LARSEN, H. \& LINDGAARD-JØRGENSEN, P. Integrated Water Resources Management in Action: Dialogue paper. Paris: UNESCO, 2009.

HERNÁNDEZ, E. Patrones de distribución de salud en la Zona Metropolitana de Toluca 2000 y 2010. México: Tesis de Licenciatura. Facultad de Geografía, UAEM, 2014.

HOLLING, C.S. The Resilience of Terrestrial Ecosystems: Local Surprise and Global Change. Sustainable Development of the Biosphere. Cambridge: WC Clark, and RE Munn, 1986, Vol. 14, p. 292-320.

JOHNSON, N. Simply Complexity: A clear guide to complexity theory. Oxford: Oneworld, 2011. 
KAIKA, M. City of flows: Modernity, nature, and the city. New York: Routledge, 2005.

KEIL, R., \& YOUNG, D. Water, Suburbs and Development: Unearthing the Toronto Region's Water Regime. Toronto: Faculty of Environment Studies, Research Development Fund, 2001.

KORENFELD FEDERMAN, D. Cultura del Agua. Hacia un uso eficiente del recurso vital. Toluca: Gobierno del Estado de México, 2009. Disponible en Internet:

https://issuu.com/ccvm/docs/cultura-del-agua-ok_web-1-91

KENNEDY, C.; CUDDIHY, J. \& ENGEL-YAN, J.A. The changing metabolism of cities. Journal of industrial ecology, 2007, Vol. 11, N², p. 43-59.

KENNEDY, C.; PINCETL, S. \& BUNJE, P. The study of urban metabolism and its applications to urban planning and design. Environmental pollution, 2011, Vol. 159, № 8, p. 1965-1973.

LEY DE AGUAS NACIONALES (LAN). Diario Oficial de la Federación. México. Publicada el 24/03/2016.

LARSIMONT, R. \& GROSSO, V. Aproximación a los Nuevos Conceptos Híbridos para Abordar las Problemáticas Hídricas. Cardinalis, 2014, Vol. 2, № 2, p. 27-48.

LINTON, J. Is the hydrologic cycle sustainable? A historical-geographical critique of a modern concept. Annals of the Association of American Geographers, 2008, Vol. 98, № 3, p. 630-649.

LINTON, J. What is water?: The history of a modern abstraction. Vancouver: UBC Press, 2010.

LINTON, J. \& BUDDS, J. The hydrosocial cycle: Defining and mobilizing a relational-dialectical approach to water. Geoforum, 2014, Vol. 57, p. 170-180.

MARTÍNEZ-AUSTRIA, P.F. y VARGAS-HIDALGO, A. Modelo dinámico adaptativo para la gestión del agua en el medio urbano. Tecnología y Ciencias del Agua, 2016, Vol. 7, N 4, p. 139-154.

MARTÍNEZ, S.E. y CASTILLO, G.H. La intermunicipalidad como modelo de gobierno local para zonas metropolitanas. En: SANTANA JUÁREZ, M.V.; HOYOS CASTILLO, G., SANTANA CASTAÑEDA, G.; PINEDA JAIMES, N.B. \& CAMPOS ALANÍS, H. Desafíos de las metrópolis: Efectos ambientales y sociales. Tendencias geográficas II. Toluca: Universidad Autónoma del Estado de México, 2016, p 33-53.

MOLLE, F. La gestion de l'eau et les apports d'une approche par la political ecology. In: Environnement, discours et pouvoir. Paris: Quae, 2012, p. 219-240.

MUNDIAL, Banco. Gestión integral de aguas urbanas. World Bank, Washington, 2012.

ORTIZ, C.R.F.; DÍAZ-DELGADO, C. y TÉLLEZ, M.H. Demanda hídrica urbana en Mexico: modelado espacial con base en sistemas de información geográfica. Interciencia, 2013, Vol. 38, №1, p. 17-25. PROGRAMA DE INDICADORES DE GESTIÓN DE ORGANISMOS OPERADORES (PIGOO). Indicadores de Gestión. Jiutepec: Instituto Mexicano de Tecnología del Agua, 2015. Disponible en internet: http://www.pigoo.gob.mx/ 
RASKIN, P. World lines: Pathways, pivots and the global future. Global Transition Initiative (GTI). Boston, Mass.: Paper Series, Tellus Institute, 2006. Disponible en Internet: www.gtinitiative.org

RIDOLFI, E. Exploring the urban hydrosocial cycle in tourist environments. Investigaciones Geográficas, 2014, № 61, p. 17-38.

SALGADO, R.; JIMENEZ, P. y CALDERÓN, J. Expansión urbana mediante la fragmentación y segregación habitacional en la Zona Metropolitana de Toluca. En: SANTANA JUÁREZ, M.V.; HOYOS CASTILLO, G., SANTANA CASTAÑEDA, G.; PINEDA JAIMES, N.B. \& CAMPOS ALANÍS, H. Desafíos de las metrópolis: Efectos ambientales y sociales. Tendencias geográficas II. Toluca: Universidad Autónoma del Estado de México, 2016. p 193-210.

SECRETARÍA DE DESARROLLO SOCIAL (SEDESOL), CONSEJO NACIONAL DE POBLACIÓN (CONAPO) E INSTITUTO NACIONAL DE ESTADÍSTICA Y GEOGRAFÍA (INEGI). Delimitación de las zonas metropolitanas de México. México: CONAPO-SEDESOL-INEGI, 2010.

SECRETARÍA DE DESARROLLO SOCIAL (SEDESOL). Expansión de las ciudades 1980-2010. México: SEDESOL, 2012.

SOBRINO, J.; GARROCHO, C.; GRAIZBORD, B.; BRAMBILA, C. \& AGUILAR, G. A. Sustainable cities: a conceptual and operational proposal. Ciudad de Panamá: United Nations Population Fund, 2015.

SWYNGEDOUW, E. Modernity and hybridity: nature, regeneracionismo, and the production of the Spanish waterscape, 1890-1930. Annals of the Association of American Geographers, 1999, Vol. $89, N^{\circ} 3$, p. $443-465$.

SWYNGEDOUW, E.; KAIKA, M. \& CASTRO, E. Urban water: a political-ecology perspective. Built Environment, 2002, Vol. 28, № 2, p. 124-137.

SWYNGEDOUW, E. Social power and the urbanization of water: flows of power. Oxford: Oxford University Press, 2004.

SWYNGEDOUW, E. Dispossessing H2O: The contested terrain of water privatization. Capitalism Nature Socialism, 2005, Vol. 16, №1, p. 81-98.

VILLAGÓMEZ-VELÁZQUEZ, Y.; AMOROZ-SOLAEGUI, I. y GÓMEZ-MARTÍNEZ, E. Los recursos hídricos en las regiones indígenas de México. Zamora Michoacán: El Colegio de Michoacán, 2013.

VON BERTALANFFY, L. The history and status of general systems theory. Academy of Management Journal, 1972, Vol. 15, № 4, p. 407-426.

WEICHSELGARTNER, J. \& KELMAN, I. Geographies of resilience Challenges and opportunities of a descriptive concept. Progress in Human Geography, 2015, Vol. 39, Nº 3, p. 249-267.

WITTFOGEL, K.A. Oriental Despotism: A Comparative Study of Total. Power. New Haven, CT: Yale University, 1957. 
WOLMAN, A. The metabolism of cities. Scientific American, 1965, Vol. 213, № 3, pp. 179-190.

ZEIGLER, B. P. Multifacetted modelling and discrete event simulation. San Diego: Academic Press Professional, Inc., 1984.

ZICCARDI, A. Participación ciudadana y políticas sociales del ámbito local. México: UNAM-Instituto de Investigaciones Sociales/Instituto Nacional de Desarrollo Social/Consejo Mexicano de Ciencias Sociales, 2004. 